\title{
Direct Simulations of 2D Fluid-Particle Flows in Biperiodic Domains
}

\author{
B. Maury
}

\begin{abstract}
We propose a method to simulate the motion of $2 \mathrm{D}$ rigid particles in a viscous, incompressible fluid. Within the Arbitrary Lagrangian Eulerian framework, momentum equations for both the fluid and the particles are discretized, and a coupled variational formulation is established. By introducing an appropriate Finite Element approximation, a symmetric linear system is obtained. This system is solved by an inexact Uzawa algorithm.

The main interest of such simulations lies in the average behaviour of a high number of particles. We therefore introduced a biperiodic formulation of the problem, which makes it possible to represent many-body mixtures at a reasonable computational cost. In order to model realistic situations, an extra term must be added to the pressure. This extra term is shown to be the lagrange multiplier associated with the vertical volume conservation constraint. We developed an appropriate unstructured mesh generator, so that the biperiodicity of the fields can be treated in a strong sense.

The question of particle contact is addressed, and a simple technique to overcome numerical problems is proposed.

The influence of periodic lengths is investigated through different simulations. The same case is simulated for different sizes of the window, and the behaviour of some space-averaged quantities is studied.
\end{abstract}

\section{Introduction}

Particulate flows arise in a wide class of industrial processes. Depending on the area of application, one can be interested in particle distribution (e.g. in lubricated transport), rate of mixing (catalyst cracking), average velocities (sedimentation columns). As the number of particles actually involved in such processes is very high, and as a deterministic description of the particle motion is not needed, the natural framework to deal with such problems is multiphase flow modeling. In Batchelor [1] (see also Prosperetti et al. [16]), probability densities are introduced in order to establish equations on averaged quantities. Depending on the situation which is studied, some models must be added in order to obtain a closed set of equations. Direct simulations of mixtures can be helpful to establish and validate those models. Especially when the flow is linear, the space of unknowns can be reduced to particle degrees of freedom, making it possible to simulate large numbers of particles. For Stokesian flows, Durlofsky et al. [4] and Dratler et al. [3] present dynamic simulations of large numbers of rigid spheres, leading to global properties of the mixture in good agreement with measurements. Similarly, for inviscid potential flows, it is possible 
to model the behaviour of high numbers of particles (see for example Sangani et al. [18]). When both viscous and inertia effects cannot be neglected, multiphase modeling lacks suitable interaction models, as pointed out by $\mathrm{Hu}$ [7]. It motivated the recent research activity on fluid-particle "full" simulations, where both particle and fluid velocities are computed.

From the numerical point of view, particulate flows are close to free surface flows: both the fluid velocity field and the domain in which it is defined are unknown. Such problems motivated the development of numerous algorithms, which can be classified in two types. The first approach is Eulerian: it is based on a fixed mesh which covers the whole domain where the fluid may be present (see Sato et al. [19] for a presentation of this method in the context of free surface flows). For particulate flows, Glowinski et al. [6] developed a fictitious domain approach. The whole domain is covered by a cartesian grid, on which local meshes are moving, following the motion of particles. The rigid motion within each particle is considered as a constraint which is imposed by a Lagrange multiplier.

Methods of the second type are based on a moving mesh which follows the motion of the fluid boundary (Lagrangian behaviour). As the mesh motion within the fluid can be defined arbitrarily, these methods are usually referred to as Arbitrary Lagrangian Eulerian. ALE methods have been applied to fluid-particle flows by Hu [7] (see also Johnson et al. [9] for $3 \mathrm{D}$ computations of spheres falling in a tube filled with liquid). $\mathrm{Hu}$ developed a finite element solver in domains which are periodic in one dimension. The non-linear system obtained by discretization of the momentum equations is solved by a Newton's algorithm. At each iteration of the algorithm, the non-symmetric sytem is solved by a GMRES method.

Following the ALE approach, we propose to apply the method of characteristics to take into account the advection term for the fluid, so that the time discretized problem turns out to be a generalized Stokes problem. A suitable variational formulation is then introduced, leading to a symmetric system which involves all the unknowns. This system is solved by a Uzawa algorithm of the Conjugate Gradient type. We present a new method to generate non-uniform biperiodic unstructured meshes of domains with holes. Such meshes make it possible to use biperiodic Finite Element spaces, so that it is not necessary to consider the biperiodicity as an extra constraint.

\section{Numerical scheme}

\subsection{Continuous equations}

Our purpose is to compute the unsteady flow of $N$ identical particles of mass $m$ in a fluid of density $\rho$ and viscosity $\mu$. We denote by $\Omega(t)$ the domain occupied by the fluid at time $t \in[0, T]$, and by $\Omega_{i}(t)$ the circle of center $\mathbf{G}_{i}$, which is the location of the $i$-th particle. The external force acting on the mixture is limited to gravity. In order to eliminate the body forces in the fluid part, we introduce the reduced pressure

$$
p=\text { physical pressure }- \text { hydrostactic pressure. }
$$


We denote by $\tilde{m}$ the excess mass of a particle (difference between the mass $m$ and the mass of fluid occupying the same volume), so that the final balance between weight and buoyant force is $\tilde{m} \mathbf{g}$. The total stress tensor is

$$
\sigma(\mathbf{x}, t)=\mu\left(\nabla \mathbf{u}+{ }^{t} \nabla \mathbf{u}\right)-p \mathbf{I}=2 \mu \mathbf{D}(\mathbf{u})-p \mathbf{I} .
$$

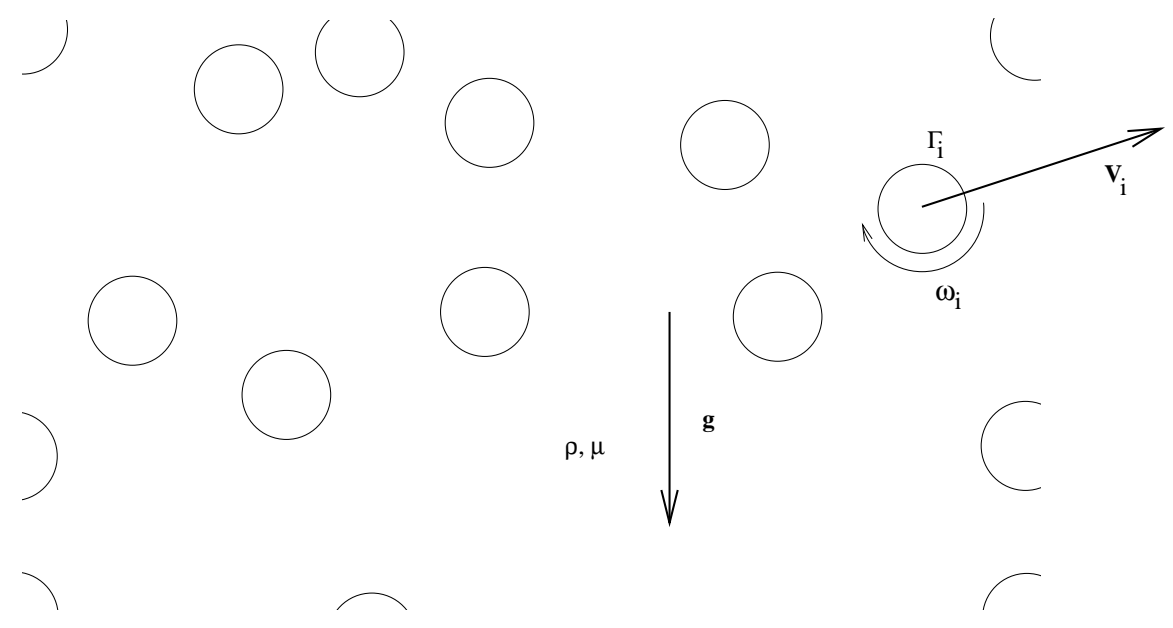

Figure III.1: Notations.

The fluid obeys the incompressible Navier-Stokes equations in $\Omega(t)$

$$
\left\{\begin{aligned}
\rho\left(\frac{\partial \mathbf{u}}{\partial t}+\mathbf{u} \cdot \nabla \mathbf{u}\right)-\nabla \cdot \sigma & =0 \\
\nabla \cdot \mathbf{u} & =0
\end{aligned}\right.
$$

while translational velocities $\mathbf{V}_{i}$ and angular velocities $\omega_{i}$ of the particles satisfy

$$
\begin{gathered}
m \frac{d \mathbf{V}_{i}}{d t}=-\int_{\Gamma_{i}(t)} \sigma \cdot \mathbf{n}+\tilde{m} \mathbf{g}, \\
J \frac{d \omega_{i}}{d t}=-\int_{\Gamma_{i}(t)}\left(\mathbf{x}-\mathbf{G}_{i}\right) \times \sigma \cdot \mathbf{n},
\end{gathered}
$$

where $J$ is the moment of inertia of a particle. The no-slip condition on the particle surface is

$$
\mathbf{u}(\mathbf{x}, t)=\mathbf{V}_{i}+\omega_{i} \times\left(\mathbf{x}-\mathbf{G}_{i}\right) \quad \forall \mathbf{x} \in \Gamma_{i}(t) .
$$

The particle distribution and the fields $\mathbf{u}$ and $p$ are supposed to be biperiodic with respect to the computational domain, as represented in Fig. III.1.

\subsection{ALE formulation}

We now introduce the Arbitrary Lagrangian-Eulerian formulation of this system (for more details, see Hughes et al. [8], Hu [7], Maury [11]). 
We first define $\mathbf{c}_{t}$, the instantaneous velocity of the domain at time $t$. It is defined on the particles by

$$
\forall x \in \Gamma_{i}, \quad \mathbf{c}_{t}(x)=\mathbf{V}_{i}+\omega_{i} \times\left(\mathbf{x}-\mathbf{G}_{i}\right),
$$

and inside the domain $\Omega(t)$ by (for example)

$$
\triangle \mathbf{c}_{t}=0
$$

Note that, when the particles are circular, the velocity of the domain on particle $i$ can be defined simply as $\mathbf{V}_{i}$ : the mesh will be allowed to slip on the particles.

The 3D field $\left(\mathbf{c}_{t}(x, t), 1\right)$ can be integrated in the physical space-time domain $S$ corresponding to the time interval $[0, T]$. From equation (6), this field is tangent to the "lateral" boundary of $S$ (boundary of $S$ excepting $\partial \Omega^{0} \times\{0\}$ and $\partial \Omega^{T} \times\{T\}$ ). It leads to mappings between the different domains $\Omega(t)$ :

$$
\begin{aligned}
\Omega\left(t_{1}\right) & \longrightarrow \Omega\left(t_{2}\right) \\
x_{1} \in \Omega\left(t_{1}\right) & \longmapsto x_{2}=C\left(x_{1}, t_{1} ; t_{2}\right)
\end{aligned}
$$

where $\left(C\left(x_{1}, t_{1} ; t\right), t\right)$ is the characteristic curve from $\left(x_{1}, t_{1}\right)$ to $\left(x_{2}, t_{2}\right)$ in $\mathrm{S}$ :

$$
\left\{\begin{aligned}
\frac{d}{d t}\left[C\left(x_{1}, t_{1} ; t\right), t\right] & =\left[\mathbf{c}_{t}(C), 1\right] \\
C\left(x_{1}, t_{1} ; t_{1}\right) & =\left(x_{1}, t_{1}\right) .
\end{aligned}\right.
$$

For each $\tau$, the $A L E$ velocity is then defined by

$$
\mathbf{u}_{\tau}(x, t)=\mathbf{u}(C(x, \tau ; t), t)
$$

with $x \in \Omega(\tau)$ and $C(x, \tau ; t) \in \Omega(t)$, which is equivalent to

$$
\mathbf{u}_{\tau}(C(x, t ; \tau), t)=\mathbf{u}(x, t) .
$$

The partial time derivation in time of (11) at $t=\tau$ gives

$$
\begin{aligned}
\frac{\partial \mathbf{u}}{\partial t} & =\frac{\partial \mathbf{u}_{\tau}}{\partial t}+\left.\frac{\partial C}{\partial t}\right|_{t=\tau} \nabla \mathbf{u}_{\tau} \\
& =\frac{\partial \mathbf{u}_{\tau}}{\partial t}-\mathbf{c}_{\tau} \nabla \mathbf{u}_{\tau}
\end{aligned}
$$

Introducing variables $\mathbf{u}_{\tau}$ and $p_{\tau}$, the Navier Stokes equations at $t=\tau$ become:

$$
\left\{\begin{aligned}
\rho\left(\frac{\partial \mathbf{u}_{\tau}}{\partial t}+\left(\mathbf{u}_{\tau}-\mathbf{c}_{\tau}\right) \cdot \nabla \mathbf{u}_{\tau}\right)-\mu \triangle \mathbf{u}_{\tau}+\nabla p_{\tau} & =0 \\
\nabla \cdot \mathbf{u}_{\tau} & =0
\end{aligned}\right.
$$

A first order form of the ALE Navier-Stokes equations (13), with $t$ in the neighbourhood of $\tau$, will be used to discretize in time equation (2). 


\section{$2.3 \quad$ Time discretization}

The time step $t^{m+1}-t^{m}$ is denoted by $\delta t$. The nonlinear term in the momentum equation is approximated by a method of characteristics (see Pironneau et al. [15]). The principle of the method is the following: $\mathbf{x}$ being the location of a fluid particle at time $t^{m+1}=t^{m}+\delta t$, and $X^{m}(\mathbf{x})$ being the location of this particle at the previous time step $t^{m}$, the total derivative at $\mathbf{x}$ is approximated by the quantity

$$
\frac{D \mathbf{u}}{D t} \approx \frac{\mathbf{u}\left(\mathbf{x}, t^{m+1}\right)-\mathbf{u}\left(X^{m}(\mathbf{x}), t^{m}\right)}{\delta t} .
$$

This method can be used within the ALE framework, as shown in Maury [11]. The only difference with the fixed domain case is that the convecting field is no longer the fluid velocity, but the relative fluid velocity $\mathbf{u}-\mathbf{c}_{t}$. The equations for the particles (3)-(4) are simply discretized by a finite difference scheme.

We denote by $\mathbf{u}^{m+1}(\cdot)$ and $p^{m+1}(\cdot)$ approximations of the fields $\mathbf{u}_{t^{m}}\left(\cdot, t^{m+1}\right)$ and $p_{t^{m}}\left(\cdot, t^{m+1}\right)$. Both $\mathbf{u}^{m+1}(\mathbf{x})$ and $p^{m+1}(\mathbf{x})$ are defined for $\mathbf{x} \in \Omega^{m}$, but correspond to the physical time $t^{m+1}$. The stress tensor at time $t^{m+1}$ is denoted by

$$
\sigma^{m+1}=\mu\left(\nabla \mathbf{u}^{m+1}+{ }^{t} \nabla \mathbf{u}^{m+1}\right)-p^{m+1} \mathbf{I}
$$

Let $\alpha$ be the reciprocal of the time step $\delta t$. The global time discretization scheme is:

$$
\begin{aligned}
\alpha \rho \mathbf{u}^{m+1}(\mathbf{x})-\mu \triangle \mathbf{u}^{m+1}(\mathbf{x})+\nabla p^{m+1}(\mathbf{x}) & =\alpha \rho \mathbf{u}^{m}\left(X^{m}(\mathbf{x})\right) \quad \text { in } \Omega^{m} \\
\alpha m\left(\mathbf{V}_{i}^{m+1}-\mathbf{V}_{i}^{m}\right) & =-\int_{\Gamma_{i}^{m}} \sigma^{m+1} \cdot \mathbf{n}+\tilde{m} \mathbf{g}, \\
\alpha J\left(\omega_{i}^{m+1}-\omega_{i}^{m}\right) & =-\int_{\Gamma_{i}^{m}}\left(\mathbf{x}-\mathbf{G}_{i}\right) \times \sigma^{m+1} \cdot \mathbf{n},
\end{aligned}
$$

with the constraint

$$
\mathbf{u}^{m+1}=\mathbf{V}_{i}^{m+1}+\omega_{i}^{m+1} \times\left(\mathbf{x}-\mathbf{G}_{i}\right), \quad \forall \mathbf{x} \in \Gamma_{i}^{m} .
$$

\subsection{Variational formulation}

In this section the $(m+1)$-upperscripts are dropped:

$$
\begin{array}{lll}
\mathbf{u}^{m+1} \longrightarrow \mathbf{u} \quad p^{m+1} \longrightarrow p & \mathbf{V}_{i}^{m} \longrightarrow \mathbf{V}_{i} \quad \omega_{i}^{m+1} \longrightarrow \omega_{i}, \\
& \sigma^{m+1} \longrightarrow \sigma \quad \Omega^{m+1} \longrightarrow \Omega,
\end{array}
$$

and the inertia and body force terms are denoted by

$$
\mathbf{f}_{\mathbf{u}}(\mathbf{x})=\alpha \rho \mathbf{u}^{m}\left(X^{m}(\mathbf{x})\right) \quad, \quad \mathbf{f}_{\mathbf{V}_{i}}=\alpha m \mathbf{V}_{i}^{m}+\tilde{m} \mathbf{g}, \quad \mathbf{f}_{\omega_{i}}=\alpha J \omega_{i}^{m} .
$$

We introduce the Hilbert space $\mathcal{V}$ consisting of all those biperiodic vector-valued functions U such that

$$
\mathbf{U}=\left(\mathbf{u}, \mathbf{V}_{1}, . ., \mathbf{V}_{N}, \omega_{1}, . ., \omega_{N}\right) \in H^{1}(\Omega)^{2} \times \mathbb{R}^{3 N}
$$


and satisfying the kinematic condition (5). The space $\mathcal{V}$ is the natural space for the velocity of the mixture. $H^{1}(\Omega)^{2}$ corresponds to the $2 \mathrm{D}$ velocity field in the fluid part, and $\mathbb{R}^{3 N}$ stands for the velocities of the particles: translational velocity (2 components for each particle), and rotational velocity (1 for each particle).

For simplicity $L_{0}^{2}$ (set of $L^{2}$ functions with zero mean value), which is the natural space for the pressure, is denoted by $Q$. The space $V$ is a Hilbert space with the scalar product

$$
\left(\mathbf{U}^{1}, \mathbf{U}^{2}\right)_{V}=\int_{\Omega} \mathbf{u}^{1} \cdot \mathbf{u}^{2}+\int_{\Omega} \nabla \mathbf{u}^{1} \cdot \nabla \mathbf{u}^{2}+\sum_{1 \leq i \leq N} \mathbf{V}_{i}{ }^{1} \cdot \mathbf{V}_{i}{ }^{2}+\sum_{1 \leq i \leq N} \omega_{i}{ }^{1} \omega_{i}{ }^{2}
$$

The system (16) becomes

$$
\begin{aligned}
\alpha \rho \mathbf{u}-\mu \triangle \mathbf{u}+\nabla p & =\mathbf{f}_{\mathbf{u}} \quad \text { in } \Omega \\
\alpha m \mathbf{V}_{i} & =\mathbf{f}_{\mathbf{V}_{i}}-\int_{\Gamma_{i}} \sigma \cdot \mathbf{n}, \\
\alpha J \omega_{i} & =\mathrm{f}_{\omega_{i}}-\int_{\Gamma_{i}}\left(\mathbf{x}-\mathbf{G}_{i}\right) \times \sigma \cdot \mathbf{n},
\end{aligned}
$$

To establish the variational formulation, we now consider a test function

$$
\tilde{\mathbf{U}}=\left(\tilde{\mathbf{u}}, \tilde{\mathbf{V}}_{1}, . ., \tilde{\mathbf{V}}_{N}, \tilde{\omega}_{1}, . ., \tilde{\omega}_{N}\right) \in V .
$$

Equation (25) is multiplied by $\tilde{\mathbf{u}}$ and integrated over $\Omega$ :

$$
\alpha \rho \int_{\Omega} \mathbf{u} \cdot \tilde{\mathbf{u}}+2 \mu \int_{\Omega} \mathbf{D}(\mathbf{u}): \mathbf{D}(\tilde{\mathbf{u}})-\int_{\Omega} p \nabla \cdot \tilde{\mathbf{u}}-\sum_{1 \leq i \leq N} \int_{\Gamma_{i}}(\sigma \cdot \mathbf{n}) \cdot \tilde{\mathbf{u}}=\int_{\Omega} \mathbf{f}_{\mathbf{u}} \cdot \tilde{\mathbf{u}}
$$

As $\tilde{\mathbf{U}} \in V$ verifies the relation (5), the boundary integrals in (29) can be written

$$
\begin{aligned}
\int_{\Gamma_{i}}(\sigma \cdot \mathbf{n}) \cdot \tilde{\mathbf{u}} & =\int_{\Gamma_{i}}(\sigma \cdot \mathbf{n}) \cdot\left(\tilde{\mathbf{V}}_{i}+\tilde{\omega}_{i} \times\left(\mathbf{x}-\mathbf{G}_{i}\right)\right) \\
& =\tilde{\mathbf{V}}_{i} \cdot \int_{\Gamma_{i}} \sigma \cdot \mathbf{n}+\tilde{\omega}_{i} \int_{\Gamma_{i}}\left(\mathbf{x}-\mathbf{G}_{i}\right) \times \sigma \cdot \mathbf{n},
\end{aligned}
$$

so that, by equations (26) and (27),

$$
\int_{\Gamma_{i}}(\sigma \cdot \mathbf{n}) \cdot \tilde{\mathbf{u}}=-\alpha m \mathbf{V}_{i} \cdot \tilde{\mathbf{V}}_{i}+\mathbf{f}_{\mathbf{V}_{i}} \cdot \tilde{\mathbf{V}}_{i}-\alpha J \omega_{i} \tilde{\omega}_{i}+\mathrm{f}_{\omega_{i}} \tilde{\omega}_{i} .
$$

Equations (25) and (31) finally lead to the global formulation:

Find $(\mathbf{U}, p) \in V \times Q$ such that

$$
\begin{cases}\alpha \rho \int_{\Omega} \mathbf{u} \cdot \tilde{\mathbf{u}}+2 \mu \int_{\Omega} \mathbf{D}(\mathbf{u}): \mathbf{D}(\tilde{\mathbf{u}})-\int_{\Omega} p \nabla \cdot \tilde{\mathbf{u}} & \\ +\alpha m \sum_{1 \leq i \leq N} \mathbf{V}_{i} \cdot \tilde{\mathbf{V}}_{i}+\alpha J \sum_{1 \leq i \leq N} \omega_{i} \tilde{\omega}_{i} & \\ =\int_{\Omega} \mathbf{f}_{\mathbf{u}} \cdot \tilde{\mathbf{u}}+\sum_{1 \leq i \leq N} \mathbf{f}_{\mathbf{V}_{i}} \cdot \tilde{\mathbf{V}}_{i}+\sum_{1 \leq i \leq N} \mathrm{f}_{\omega_{i}} \tilde{\omega}_{i} & \forall \tilde{\mathbf{U}} \in V, \\ \int_{\Omega} \tilde{p} \nabla \cdot \mathbf{u}=0 & \forall \tilde{p} \in Q,\end{cases}
$$

The mass conservation is simply

$$
\int_{\Omega} \tilde{p} \nabla \cdot \mathbf{u}=0 \quad \forall \tilde{p} \in Q
$$




\subsection{Numerical solution}

We denote by $V_{h}$ and $Q_{h}$ approximation spaces for $H^{1}(\Omega)$ and $L^{2}(\Omega)$, satisfying the inf-sup condition. The computations presented at the end of the paper use the so-called minielement: the pressure is piecewise $P^{1}$, and the velocity is the sum of a piecewise $P^{1}$ field and a linear combination of bubble functions (a bubble function associated with a triangle is the product of the barycentric coordinates with respect to the 3 vertices, and is zero outside the triangle). This element is known to satisfy the inf-sup condition.

Our first step is to assemble the matrices corresponding to the decoupled problem (the kinematic condition is not taken into account). It leads to matrices $A, B^{T}, B$, algebraic analogs of the Stokes, gradient, and divergence operators, respectively. Matrix $A$ is block-diagonal (fluid-particles), and the submatrix of $A$ corresponding to the particles is diagonal. Note that $A$ will contain non-zero extra diagonal terms as soon as any triangle is in contact with 2 particles.

The approximate velocity is to be found in the subspace of $V_{h}$ satisfying the kinematic condition. The velocity is written $\mathbf{u}=\left(\mathbf{u}_{\Omega}, \mathbf{u}_{\Gamma}\right)$, where $\mathbf{u}_{\Gamma}$ contains the components of the field associated with the vertices on the particle boundaries. The no-slip condition can then be expressed in the following algebraic form:

$$
\left(\mathbf{u}_{\Gamma}\right)^{T}=\tilde{P}\left(\mathbf{V}_{1}, . ., \mathbf{V}_{N}, \omega_{1}, . ., \omega_{N}\right)^{T}
$$

or, equivalently, by

$$
\left(\mathbf{u}_{\Omega}, \mathbf{u}_{\Gamma}, \mathbf{V}_{1}, . ., \mathbf{V}_{N}, \omega_{1}, . ., \omega_{N}\right)^{T}=P\left(\mathbf{u}_{\Omega}, \mathbf{V}_{1}, . ., \mathbf{V}_{N}, \omega_{1}, . ., \omega_{N}\right)^{T}
$$

with

$$
P=\left[\begin{array}{cc}
I & \mathcal{O} \\
\mathcal{O} & \tilde{P} \\
\mathcal{O} & I
\end{array}\right]
$$

The algebraic problem can then be written

$$
\left[\begin{array}{cc}
P^{T} A P & P^{T} B^{T} \\
B P & \mathcal{O}
\end{array}\right]\left[\begin{array}{l}
\mathbf{U} \\
p
\end{array}\right]=\left[\begin{array}{l}
F \\
0
\end{array}\right]
$$

An inexact Uzawa algorithm (see Elman and Golub [5]) is performed on the reduced symmetric system

$$
B P\left(P^{T} A P\right)^{-1} P^{T} B^{T} p=B P\left(P^{T} A P\right)^{-1} F .
$$

At each iteration of the algorithm, the system $P^{T} A P \mathbf{x}=\mathbf{b}$ is solved by a conjugate gradient method, preconditioned by its diagonal $D$ :

$$
D^{-1 / 2}\left(P^{T} A P\right) D^{-1 / 2} \mathbf{x}^{\prime}=D^{-1 / 2} \mathbf{b}
$$

with $\mathbf{x}^{\prime}=D^{1 / 2} \mathbf{x}$

The global system for pressure (37) is preconditioned by a Laplace problem with Neuman boundary conditions. 
Remark: As the matrix $A$ corresponds to the generalized Stokes operator with natural boundary conditions, this technique can be immediately applied to any Finite Element discretization of the stokes problem, as soon as the solution method is iterative. For a direct method, the matrices $P^{T} A P$ and $B P$ should be assembled, which is not performed in the present approach.

\section{$2.6 \quad$ Remeshing}

As the geometry of the domain undergoes high changes during the simulation, the mesh is likely to degenerate. In order to avoid critical situations, the quality of the mesh (i.e. quality of the worst triangle in the mesh) is estimated at each time step. When this quality drops below a prescribed value, the whole domain is remeshed. As the velocity at the previous time step is needed to take into account the advection, the velocity field defined on the old mesh is projected onto the new one. The method we use to perform this projection is similar to what is presented in $\mathrm{Hu}$ [7]. In actual computations, like those presented in this paper, the domain is remeshed every 5 or 10 time steps.

\section{Biperiodic simulations}

\subsection{Model}

The biperiodic formulation obtained by a straightforward transformation of (2)-(3)-(4)-(5) does not represent a realistic physical situation. Indeed, as the force exerted on the system is constant and non-zero, the velocity of the center of mass of the considered system is unbounded: the system falls down freely. In the case of a potential flow around bubbles (no mass), it is natural to impose a zero average velocity for the fluid, and a constant pressure drop across the computational cell (see Sangani et al. [17]). Such a constraint is necessary to set up a well-posed problem. Similarly, for Stokes flows, a zero-volume-flowrate condition has to be prescribed by adding a backflow pressure gradient (see Pedercini et al. [14]). In the present case, because of inertia, the problem is well-posed, only the model is not relevant. We propose to add the fact that the "large container" which contains the mixture does have a bottom. Even if we are only interested in the behaviour of the mixture far away from this bottom, this condition is necessary to ensure volume conservation in the vertical direction.

In what follows, we will use the fact that the fluid velocity field $\mathbf{u}$ (and, similarly, the corresponding test functions $\tilde{\mathbf{u}}$ ) can be extended within the rigid particle to define a divergence-free field in the whole domain occupied by the mixture. For any $\mathbf{x} \in \Omega_{i}, \mathbf{u}(\mathbf{x})$ is simply defined by

$$
\mathbf{u}(\mathbf{x})=\mathbf{V}_{i}+\omega_{i} \times\left(\mathbf{x}-\mathbf{G}_{i}\right) .
$$

We propose to change the model in the following way: 
1. What we defined as the reduced pressure in section 2.1 (pressure - hydrostatic pressure) is biperiodic only up to a correction term of the "hydrostatic" type $\eta z$, where $\eta$ is an unknown scalar which may depend on the time. We therefore have to give a new definition of the reduced pressure. If we denote by $p^{\prime}$ the physical pressure, this new reduced pressure is

$$
p=p^{\prime}+\rho g z+\eta z
$$

which we will assume biperiodic.

2. The average vertical motion of the mixture is identically zero:

$$
\int_{\Omega_{t o t}} \mathbf{u} \cdot \mathbf{e}_{z}=0
$$

where $\Omega_{t o t}$ is the domain occupied by the fluid and the particles. Note that it differs from specifying a zero motion of the center of mass of the system, as soon as the particles and the fluid do not have the same density.

Let us show that the new variable $\eta$ introduced in Eq. (40) can be considered a Lagrange multiplier for the constraint (41). The momentum equations for the fluid and the particles become, respectively,

$$
\rho \frac{D \mathbf{u}}{D t}-\nabla \cdot \sigma+\eta \mathbf{e}_{z}=0
$$

and

$$
m \frac{d \mathbf{V}_{i}}{d t}=-\int_{\Gamma_{i}} \sigma \cdot \mathbf{n}+m \mathbf{g}+\int_{\Gamma_{i}} z \eta \mathbf{n} .
$$

The only difference with the variational formulation previously obtained is the addition of a term $\star$ to the left-hand side:

$$
\star=\eta \int_{\Omega} \mathbf{e}_{z} \cdot \tilde{\mathbf{u}}-\eta \sum_{1 \leq i \leq N} \int_{\Gamma_{i}} z \mathbf{n}_{i} \cdot \tilde{\mathbf{u}}
$$

where $\mathbf{n}_{i}$ is the normal pointing inward the particle $i$. Partial integration yields

$$
-\sum_{1 \leq i \leq N} \int_{\Gamma_{i}} \eta z \mathbf{n}_{i} \cdot \tilde{\mathbf{u}}=\eta \sum_{1 \leq i \leq N} \int_{\Omega_{i}} \mathbf{e}_{z} \cdot \tilde{\mathbf{u}}+\eta \sum_{1 \leq i \leq N} \int_{\Omega_{i}} z \nabla \cdot \tilde{\mathbf{u}}
$$

As the test functions are divergence-free within all the rigid particles ( $\tilde{\mathbf{u}}$ is defined within each particle by (39)), we finally obtain

$$
\begin{aligned}
\star & =\eta \int_{\Omega} \mathbf{e}_{z} \cdot \tilde{\mathbf{u}}+\eta \sum_{1 \leq i \leq N} \int_{\Omega_{i}} \mathbf{e}_{z} \cdot \tilde{\mathbf{u}} \\
& =\eta \int_{\Omega_{t o t}} \mathbf{e}_{z} \cdot \tilde{\mathbf{u}}
\end{aligned}
$$

which is exactly the integral defining the constraint (41), multiplied by $\eta$. The variational formulation of the new problem is then: 
Find $(\mathbf{U}, p, \eta) \in V \times Q \times \mathbb{R}$ such that

$$
\begin{cases}\alpha \rho \int_{\Omega} \mathbf{u} \cdot \tilde{\mathbf{u}}+2 \mu \int_{\Omega} \mathbf{D}(\mathbf{u}): \mathbf{D}(\tilde{\mathbf{u}})-\int_{\Omega} p \nabla \cdot \tilde{\mathbf{u}}+\eta \int_{\Omega_{t o t}} \mathbf{e}_{z} \cdot \tilde{\mathbf{u}} \\ +\alpha m \sum_{1 \leq i \leq N} \mathbf{V}_{i} \cdot \tilde{\mathbf{V}}_{i}+\alpha J \sum_{1 \leq i \leq N} \omega_{i} \tilde{\omega}_{i} & \forall \tilde{\mathbf{U}} \in V, \\ =\int_{\Omega} \mathbf{f}_{\mathbf{u}} \cdot \tilde{\mathbf{u}}+\sum_{1 \leq i \leq N} \mathbf{f}_{\mathbf{V}_{i}} \cdot \tilde{\mathbf{V}}_{i}+\sum_{1 \leq i \leq N} \mathbf{f}_{\omega_{i}} \tilde{\omega}_{i} & \forall \tilde{p} \in Q, \\ \int_{\Omega} \tilde{p} \nabla \cdot \mathbf{u}=0 & \forall \tilde{\eta} \in \mathbb{R}, \\ \tilde{\eta} \int_{\Omega_{t o t}} \mathbf{e}_{z} \cdot \mathbf{u}=0 & \end{cases}
$$

so that $\eta$ turns out to be a Lagrange multiplier for the new constraint (41), just as $p$ is a Lagrange multiplier for the incompressibility constraint. As $\eta$ is a scalar, the associated test function $\tilde{\eta}$ is a scalar as well. Space discretization of this formulation can be performed as in section 2.5. The algebraic formulation which is obtained involves the new variable $\eta$ :

$$
\left[\begin{array}{ccc}
P^{T} A P & P^{T} B^{T} & P^{T} C^{T} \\
B P & \mathcal{O} & \mathcal{O} \\
C P & \mathcal{O} & \mathcal{O}
\end{array}\right]\left[\begin{array}{c}
\mathbf{U} \\
p \\
\eta
\end{array}\right]=\left[\begin{array}{c}
F \\
0 \\
0
\end{array}\right]
$$

where $C$ is a row-matrix, which results from space discretization of $\int_{\Omega_{t o t}} \mathbf{e}_{z} \cdot \mathbf{u}$.

Remark:As the correcting pressure is the Lagrange multiplier of the homogeneous constraint, it has no effect on the energy balance. As a matter of fact, the additional force does not exert any global work on the mixture.

\subsection{Numerical solution}

The new problem might be solved by a Uzawa algorithm on the space of Lagrange multipliers $Q \times \mathbb{R}$ : the velocity is eliminated, and the symmetric definite system

$$
\left[\begin{array}{l}
B P \\
C P
\end{array}\right]\left(P^{T} A P\right)^{-1}\left[\begin{array}{ll}
P^{T} B^{T} & P^{T} C^{T}
\end{array}\right]\left[\begin{array}{l}
p \\
\eta
\end{array}\right]=\left[\begin{array}{c}
B P \\
C P
\end{array}\right]\left(P^{T} A P\right)^{-1} F
$$

is solved, as system (37) was, by a conjugate gradient method. Nevertheless, as $\eta$ is a global Lagrange multiplier, whereas $p$ is a set of local ones, it suggests a decoupled solution. Indeed, numerical experiments on the whole dual problem (50) showed bad convergence properties. We therefore propose the following algorithm, based on the preliminary computation of $\eta$. Both velocity and pressure are eliminated, which leads to

$$
\underbrace{C\left[\begin{array}{cc}
P^{T} A P & P^{T} B^{T} \\
B P & \mathcal{O}
\end{array}\right] C^{T}}_{M} \eta=C\left[\begin{array}{cc}
P^{T} A P & P^{T} B^{T} \\
B P & \mathcal{O}
\end{array}\right]\left[\begin{array}{l}
F \\
0
\end{array}\right] .
$$


Note that matrix $M$ is a $1 \times 1$ matrix. A conjugate gradient algorithm performed on system (51) therefore converges exactly in one step. Velocity and pressure are then determined by solving

$$
\left[\begin{array}{cc}
P^{T} A P & P^{T} B^{T} \\
B P & \mathcal{O}
\end{array}\right]\left[\begin{array}{l}
\mathbf{U} \\
p
\end{array}\right]=\left[\begin{array}{c}
F \\
0
\end{array}\right]-C^{T} \eta,
$$

which is exactly system (36) with an extra term added to the right-hand side.

\subsection{Biperiodic mesh generation}

In order to compute the biperiodic velocity and pressure fields, a biperiodic mesh is generated. As the domain occupied by the fluid is far from being convex, it is in general impossible to delimit the biperiodic "window" by straight lines. In this section we present a new method to generate a periodic or biperiodic mesh of a domain with holes.

Let us consider a distribution of particles consistent with the periodic geometry, as in Fig. III.2. The rectangle $R=R_{1} \cup R_{2} \cup R_{3} \cup R_{4}$ delimits the biperiodic window which is to become the computational domain. Its vertices are denoted by $A_{1}, A_{2}, A_{3}$, and $A_{4}$. The edges and the vertices are related by

$$
R_{3}=R_{1}+\overrightarrow{A_{1} A_{4}}, \quad R_{4}=R_{2}+\overrightarrow{A_{2} A_{1}} .
$$

Note that in case no particle intersects the rectangle, there is no need to develop a special procedure to generate a mesh.

\section{Strategy}

The approach consists in generating a broken closed line $\tilde{R}=\tilde{R}_{1} \cup \tilde{R}_{2} \cup \tilde{R}_{3} \cup \tilde{R}_{4}$ such that

- $\tilde{R}$ is not too far from $R$,

- $\tilde{R}$ is biperiodic: it verifies

$$
\tilde{R}_{3}=\tilde{R}_{1}+\overrightarrow{A_{1} A_{4}}, \quad \tilde{R}_{4}=\tilde{R}_{2}+\overrightarrow{A_{2} A_{1}} .
$$

- there is no contact between $\tilde{R}$ and the particles.

Such a "broken rectangle" can be used to generate a biperiodic mesh compatible with the particle distribution. The torus-type topology is then achieved by one-to-one identification of the vertices of the 2 horizontal sides $\left(\tilde{R}_{1}\right.$ and $\left.\tilde{R}_{3}\right)$, the 2 vertical sides $\left(\tilde{R}_{2}\right.$ and $\left.\tilde{R}_{4}\right)$, and the identification of the 4 corners.

\section{Construction of $\tilde{R}$}

In order to generate $\tilde{R}_{1}$, a subwindow around $R_{1}$ is defined. The height of this subwindow $W$ is a few times (typically 10) the diameter of the particles. $W$ is filled with the actual particles by periodicity, but the particles which intersect the boundary of $W$ are suppressed. The next step is then to chose a point $B_{1}$ in $W$ in the fluid part (outside the 
particles), close to $A_{1}$, the left end of $R_{1}$. We define $B_{2}=B_{1}+\overrightarrow{A_{1} A_{2}}$. A mesh of $W$ minus the remaining particles, such that $B_{1}$ and $B_{2}$ belong to the set of vertices, is then generated in a classical way. The line $\tilde{R}_{1}$ will be defined as a connection from $B_{1}$ to $B_{2}$ through the mesh.

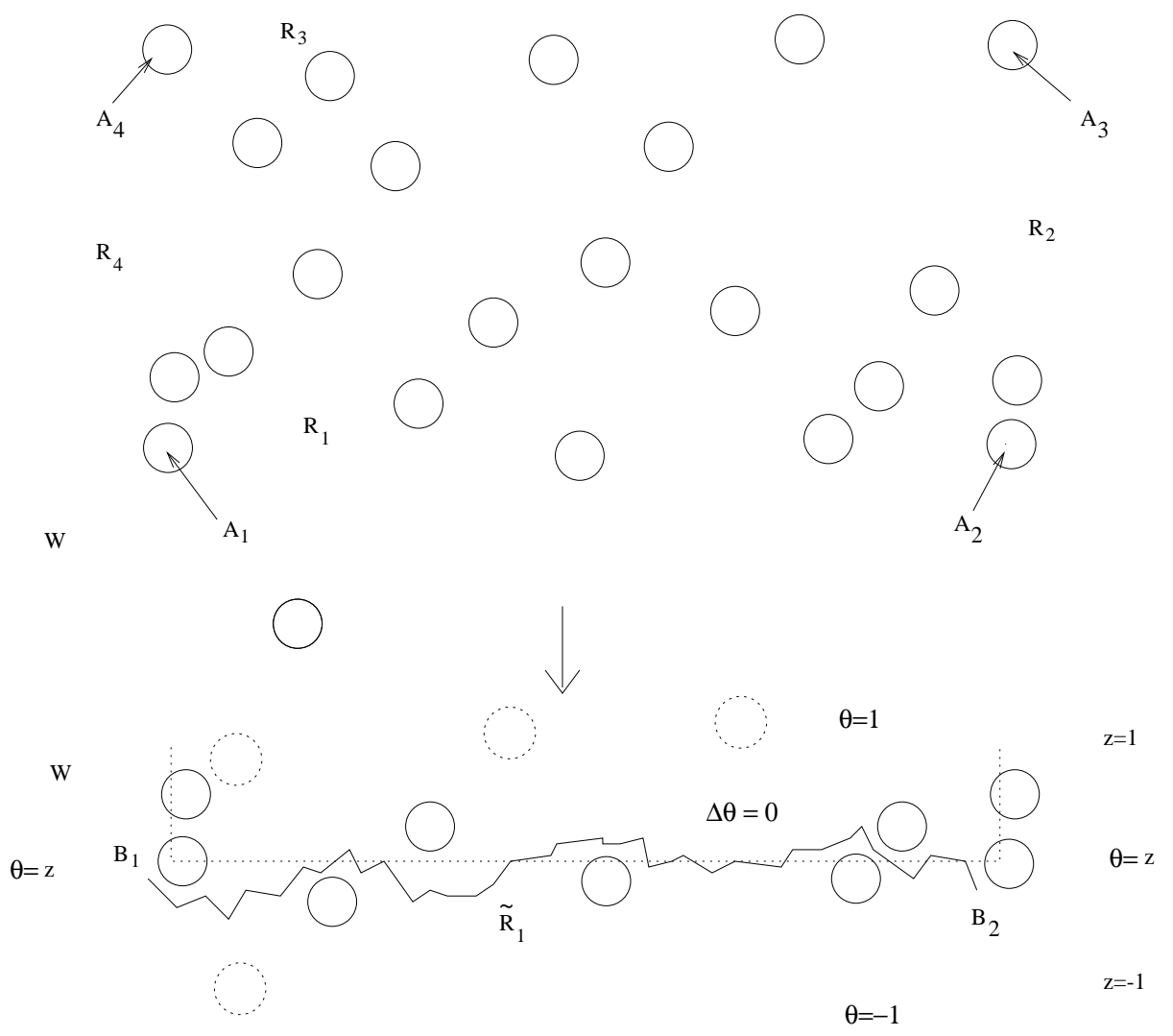

Figure III.2: Mesh generation.

In order to build this broken line we propose the following approach, which could be applied to 3D problems: a scalar field $\theta$ is introduced, solution of the Laplace equation in $W$, with uniform Dirichlet boundary conditions on each particle. We take the prescribed value on the boundary of a particle proportional to the height of its center (the height origin is set at $R_{1}$ ). A similar condition is taken for the horizontal sides of $W_{1}$, and homogeneous Neuman B.C. are prescribed on the lateral parts. Let us now consider the subset of triangles intersecting the set $\left\{x ; \theta(x)=\theta\left(B_{1}\right)=\theta\left(B_{2}\right)\right\}$. It is a submesh of the initial one, it contains $B_{1}$ and $B_{2}$, and either one of its top or bottom boundaries can be chosen as $\tilde{R}_{1}$, provided it is connected to $B_{1}$ and $B_{2}$.

A similar approach is used to build $\tilde{R}_{2}$. The two remaining broken lines $\tilde{R}_{i}$ are defined by $\tilde{R}_{3}=\tilde{R}_{1}+\overrightarrow{A_{1} A_{4}}$ and $\tilde{R}_{4}=\tilde{R}_{2}+\overrightarrow{A_{2} A_{1}}$. The final broken frame $\tilde{R}$ is then $\tilde{R}_{1} \cup \tilde{R}_{2} \cup \tilde{R}_{3} \cup \tilde{R}_{4}$.

Remark:There are other ways to generate a broken line through a domain with obstacles. Three advantages of the presented method are: 
- In case of a non-uniform mesh, the "local" meshes are built with the same $h$-distribution, so that the broken line integrates smoothly into the final non-uniform mesh.

- This method is robust, even if the solid volume fraction is high (up to 55/100).

- Although it has not yet been implemented for $3 D$ meshes, this method is applicable to them, mainly because the piecewise linear surface is defined implicitly as the boundary of a mesh, and therefore is not difficult to build.

\section{Particle "contacts"}

\subsection{Introduction}

This section adresses the problem encountered when two or more particles come to near contact. Conceptually, such a situation can be handled in the framework which has been presented, provided the finer zone between the particles is refined. Indeed, lubrication theory ensures that the interparticle distances will not vanish. Numerical experiments based on local mesh refinements (see $\mathrm{Hu}[7]$ ) show good stability and robustness properties. Nevertheless, this approach has some drawbacks:

- It is impossible to predict a priori the number of necessary refinement steps, so that there is no control on computational costs, nor on memory requirements.

- The time step has to be controlled, and may have to be reduced drastically in order to avoid overlapping of particles.

- The high non-uniformity of the computational mesh is harmfull to the conditionning of the numerical system to solve.

\subsection{Technical treatment}

\subsubsection{Principle of the method}

In this section, a "cut off"-like technique is presented. It ensures numerical stability at almost no computational cost. Although this technique seems to act as if particle roughness were taken into account, it is not based on any proper physical modeling. Some remarks on the underlying physics will be given in the next section.

Let $Y$ represent the particle configuration:

$$
Y=\left(\mathbf{G}_{1}, \ldots, \mathbf{G}_{N}, \theta_{1}, \ldots, \theta_{N}\right) .
$$

The distance between particles $i$ and $j$ is denoted by $\mathrm{D}_{i j}$, which can be considered a function of $Y$. In periodic domains, the distance between 2 particles is of course the minimum 
of distances between their "images" obtained by periodicity. The set of feasible states (particles do not touch) is

$$
\Lambda=\left\{Y=\left(\mathbf{G}_{i}, \theta_{i}\right)_{1 \leq i \leq N} \in \mathbb{R}^{3 N} \text { s.t. } \mathrm{D}_{i j}>0, \forall i, j\right\} .
$$

It is clear that any $Y \in \Lambda$ can be obtained in numerical simulations, even those with arbitrarily small $\mathrm{D}_{i j}{ }^{\prime} \mathrm{s}$. Moreover, if the time step is too large, some particles might even overlap. As we want to avoid the latter configurations, we propose to replace any problematic $Y$ by another configuration $Y_{\varepsilon}$ in

$$
\Lambda_{\varepsilon}=\left\{Y=\left(\mathbf{G}_{i}, \theta_{i}\right)_{1 \leq i \leq N} \in \mathbb{R}^{3 N} \text { s.t. } \mathrm{D}_{i j} \geq \varepsilon, \forall i, j\right\},
$$

where $\varepsilon>0$ is a fixed parameter, and $Y_{\varepsilon}$ is close to $Y$ in a certain sense. The choice of $\varepsilon$ is conditioned by numerical considerations: as a mesh has to be generated in the fluid part, it is necessary to keep finite interparticle distances. The value of $\varepsilon$ is the prescribed lower bound of interparticle distances. This value will of course affect the computed flow, but numerical tests showed that it exerts no significant influence as soon as it drops below a certain limit. In all computations, the value of $\varepsilon$ has been set to $5 \%$ of the particle diameter.

\subsubsection{Numerical implementation}

In this section we present a numerical method to compute a reasonable $Y_{\varepsilon}$ from a given $Y$, i.e. to transform a "bad" situation $Y \notin \Lambda_{\varepsilon}$ onto a "good" one $Y_{\varepsilon} \in \Lambda_{\varepsilon}$. As we want $Y_{\varepsilon}$ to be close to $Y$, it would be natural to use a projection onto $\Lambda_{\varepsilon}$. Unfortunately, as $\Lambda_{\varepsilon}$ is not convex, such a projection cannot be defined properly. We therefore propose a heuristic method to compute $Y_{\varepsilon}$, based on the minimizing of a functional. Let $\Psi$ be the real functional defined, for any configuration $Y \in \mathbb{R}^{3 N}$, by

$$
\Psi(Y)=\sum_{\mathrm{D}_{i j}<\varepsilon}\left(\mathrm{D}_{i j}(Y)-\varepsilon\right)^{2}
$$

The set $\Lambda_{\varepsilon}$ is exactly $\{Y$ s.t. $\Psi(Y)=0\} . \quad Y_{\varepsilon}$ is obtained by performing a steepest descend algorithm on $\Psi$, starting from $Y$.

At each step of the steepest descent algorithm, all the distances between particles must be estimated with accuracy, which can be very costly especially if particles are not circles. In order to reduce the number of calls of this procedure, the whole domain is covered with a coarse regular grid (whose step is larger than the diameter of the particle $+2 \varepsilon$ ). Each particle is located in this grid, and only the couples belonging to neighbouring cells are tested. In case of periodic calculations, also the "ghost" particles, not represented in the computational mesh, must be taken into account.

This method has shown its robustness in numerical tests. It makes possible long-time simulations with no risk of overlapping. Furthermore, as the minimizing procedure is performed on the space of all particle positions, it can handle close packed arrangements, where some particles may be in contact with 6 other ones. 


\subsection{Further prospects: Lubrication models}

In particular situations, e.g. when some particles are sticking to a wall (particle laying at the bottom of a container), or more generally when the solid phase is high, the lubrication forces may play a significant role on the overall behaviour of the mixture (see Dratler and Schowalter [3] for the influence of near contact dynamics on the global viscosity of suspensions). In [13], we introduced a many-body lubrication model based on a first order asymptotic expansion of the lubrication flow between two spheres developed by Kim et al. [10]. Although this method reproduces the physical behaviour properly in very simple cases (e.g. single particle approaching the bottom of a container under the action of gravity), we will not present in this paper any many-body simulation based on the coupling of the two approaches, because we are unable to define a non-trivial test case which would illustrate the accuracy of the coupled approach. Indeed, interparticle distances are very difficult to measure experimentally. Furthermore, when the interparticle gap is too thin, it may lead to film rupture, which is up to now impossible to take into account with such models.

\section{$5 \quad$ Numerical experiments}

We present here 4 sets of simulations. As this direct approach is intended to lead to a better understanding of the global behaviour of "infinite" mixtures, a special attention will be paid to the influence of the biperiodic model on the computed flow (sets II and III). Situations are characterized by the solid volume fraction $\Phi$ and the particle Reynolds number. The latter is defined by $\operatorname{Re}_{p}=\rho U d / \mu$, where $\mathrm{U}$ is the maximum modulus of the fluid velocity, and $d$ is the particle diameter. The time step is controlled automatically in all the simulations. The control is based on a particle CFL condition. It is chosen so that the maximum motion of a particle between two steps is its diameter multiplied by a constant factor 0.3 .

\subsection{Non-circular particles}

The first set of pictures illustrates the suitability of the method to general situations: the motion of 1000 ellipses of different sizes is computed. The volume fraction is around 1/100, and the particle Reynolds number is 10 .

Fig. III.3a : Boundary of the mesh (boundary of the periodic window + particles).

Fig. III.3b: Mesh corresponding to the selected zone in Fig III.3b.

Fig. III.4: Computed velocity field (detail). 


\subsection{Periodic model}

This set of pictures illustrates the problem of the biperiodic window size. Figures III.5a and III.5b correspond to a 2000 particles computation. The number of nodes of the triangulation is about $10^{5}$. The solid volume fraction $\Phi$ is $15 / 100$, the periodic length is $L=6$, and $\operatorname{Re}_{p}=5$. We will refer to it as Large Window simulation (LW). In Fig. III.5a, only the particle velocities are represented. Although it does not represent a continuous field, it gives a good overview of the global motion of the mixture. Fig. III.5b shows the fluid velocity field in the zone delimited by the square in Fig. III.5a. The field which is represented is of course not biperiodic.

The next pictures correspond to the very same physical situation: the size of the particles, the solid fraction $\Phi$, the fluid properties, the body forces, are unchanged. The only difference is the size of the window, which is reduced to the zoom represented in the previous figure (4 times smaller, $L=1.5$ ). The number of particles is now 2000/16 $=125$. We will call it the Small Window simulation (SW).

The influence of the biperiodicity constraint already appears in the velocitiy fields (Figs. III.5a and III.6a). The large structures observed in the LW simulation can obviously not develop in the SW.

The better way to estimate the suitability of the model would consist in extracting a subdomain $1.5 \times 1.5$ out of the large domain $6 \times 6$, and compare the behaviour of the mixture to the behaviour obtained with the $1.5 \times 1.5$ direct biperiodic simulation. Such a comparison cannot be done straightforwardly, because $\Phi$ is likely to vary in the artificial subdomain. For example, the kinetic energy of the solid phase will undergo a jump as soon as a new particle comes into the subdomain. This problem should be overcome in the future, either by introducing more sophisticated filters which take into account the variations of $\Phi$, or by increasing the number of particles.

We will limit here the analysis of those simulations to some various kinds of kinetic energy which can be associated with particle velocities. For both simulations, we represent the total kinetic energy of the solid phase $E_{\text {tot }}$, the kinetic energy associated with the mean velocity of the solid phase $E_{\text {macro }}$, and the kinetic energy of a single particle $E_{\text {single }}$. Those quantities are scaled in such a way that a constant and uniform motion of the set of particles would lead to three identical values. Those functions of time are represented in Figs. III.7a and III.7b.

Firstly, an important remark can be made for both simulations. There is a huge relative difference between $E_{\text {tot }}$ and $E_{\text {macro }}$, which expresses the fact that the motion is completely different from a uniform translation. The quantity $E_{\text {tot }}-E_{\text {mean }}$ can be seen as a temperature of the solid phase. In the present situation, one can consider that most of the potential energy deteriorates into viscous dissipation and particle temperature.

Considering now the differences between the two graphs, it is clear that the total kinetic energy behaves more steadily in the LW simulation, as the next set of simulations will confirm. 
Finally, the energetic history of a single particle seems to be dominated by characteristic frequencies varying from a case to the other. Indeed, high frequencies which dominate in SW are still observable in LW but are no longer predominating. A systematic study of this phenomenon still has to be performed.

\subsection{Global steady state}

The next set of figures addresses the following question: Does the mean flow behave steadily if the window size is large enough? A given set of physical parameters are chosen, and three simulations are performed on different window sizes. The value of $\Phi$ is $14 / 100$ and the particle Reynolds number is $\operatorname{Re}_{p}=4$. The evolution of average $x-$ and $z$-velocities of the fluid and the particles are represented on Figs. III.8a, III.8b and 8c. Figs. III.9a, III.9b and III.9c show the time-derivatives of the different energies: kinetic, potential, dissipated, and the sum of them all. The singularities appearing in the kinetic energy curve are purely numerical, they correspond to time steps at which a remeshing has been performed. A better remeshing-projection scheme has to be developed. Nevertheless, those singularities seem to be localized in time, and they do not prevent us from analyzing the kinetic energy evolution.

The first observation lies in the time averaged quantities. All quantities oscillate around a constant value which does not depend significantly on the window size. The small window simulation appears to be sufficient to predict the mean sedimentation velocity for example, provided a time average is performed.

Secondly, the flow seems to stabilize globally as the periodic length is increasing. Nevertheless, it is to be noticed that, even for the highest number of particles $(N=800)$, some fluctuations can still be observed at some times of the computation (see for example the time derivative of kinetic energy for $N=800$ around time $t=50)$. It must be added that, in all the long-time simulations we performed, such phenomena occurred. Indeed, for large periodic lengths, average quantities are almost always constant, but undergo high perturbations at certain times. Those perturbations can be associated with a special pattern of the mixture flow: a large eddy develops all over the computational domain, increasing global kinetic energy. This phenomenon might be due to the biperiodicity of the domain. Indeed, as soon as many particles constitute an aggregate whose size is close to the vertical periodic length, this aggregate has to be considered with all its periodic images: it acts like a vertical chain of macro-bodies. No significant force is exerted by the fluid on such a vertical chain, so that the mean velocity of the aggregate increases, inducing a macro-eddy on the computational domain. In real flows, it is known that such a vertical configuration is not stable. Any vertical chain tends to collapse before it reaches a high velocity. To the contrary, in our computations, the vertical chain is stable, because the periodicity prevents the chain from collapsing. Some numerical tests still have to be performed to verify this explanation. If it is valid, those phenomena should disappear as soon as the gravity acts in a direction which is no longer a direction of periodicity. 


\subsection{Global interaction force}

As a first step toward general models, we shall finally present an example of what the biperiodic direct approach can provide. We will concentrate on a single relation on which most two-phase macroscopic models are based, namely the dependency between interaction force and relative velocity.

\subsubsection{Space-time averaging}

The fluid particle interaction force is not computed explicitely in the algorithm. In order to avoid extra computations of boundary integrals, we propose here a simple way to estimate the mean interaction force and to relate it with the mean relative velocity.

Let us consider a given situation (size of the particles, densities, viscosity, solid fraction). Let $\bar{V}_{f}$ be the mean (space averaged) vertical velocity of the fluid, $\bar{V}$ the mean vertical velocity of the particles (average over all of them), and $\bar{F}$ the mean force exerted by the fluid per particle. Our purpose in this section is to investigate the relation between the time-averaged quantities $\left\langle\bar{F}>\right.$ and $\left\langle\bar{V}-\bar{V}_{f}>\right.$.

For any value of the gravity modulus $|\mathbf{g}|$, the mean vertical velocity $\bar{V}$ of the particles (average over all of them) can be represented as a function of time, as in the set of figures III.8. As we previously noticed, after a transitory phase, this quantity oscillates around a constant value. This function of time can be averaged over a few periods of oscillation to give a good representation of the mean velocity $\langle\bar{V}\rangle$. Besides, when the system has reached a pseudo steady state, the kinetic energy oscillates around a constant value, so that hydrodynamic forces balance exactly (up to oscillating terms) the weight of the particles, which is known:

$$
<\bar{F}>=-m \mathbf{g} .
$$

Furthermore, as the mean vertical velocity of the mixture is zero, it comes

$$
\Phi<\bar{V}>+(1-\Phi)<\bar{V}_{f}>=0
$$

so that the mean relative velocity can be expressed

$$
<\bar{V}-\bar{V}_{f}>=\frac{1}{(1-\Phi)}<\bar{V}>
$$

\subsubsection{Numerical simulations}

Using equations (59) and (61), we can now associate with any long-time simulation (for which $|\mathbf{g}|$ and $\langle\bar{V}>$ are known) a point in the relative velocity - interaction force plane. We present here 3 sets of simulations, corresponding to 3 different values of $\Phi: 3.10^{-3}$, 0.112 , and 0.28 ( $N=1, N=40$ and $N=100$, respectively). 
The periodic length $L=1$ is the same for all simulations, the particle radius is 0.03 , and the viscosity of the fluid is $5 \cdot 10^{-4}$. The particle Reynolds number depends of course on the prescribed gravity. It varies between 5 and 360. Figs. III.10a, III.10b and III.10c show the velocity fields corresponding to $\operatorname{Re}_{p}=5, \operatorname{Re}_{p}=140$, and $\operatorname{Re}_{p}=360$. Although it is questionable to represent non-divergence free fields, we chose to represent the fluid velocity relatively to the mesh velocity, i.e. $\mathbf{u}-\mathbf{c}$, because they illustrate more properly the differences between the Stokes flow $\left(\operatorname{Re}_{p}=5\right)$ and the Navier-stokes flows $\left(\operatorname{Re}_{p}=140\right.$ and $\left.\operatorname{Re}_{p}=360\right)$.

For each set of simulation (i.e. for each value of $\Phi$ ), the curve

$$
\bar{V}-\bar{V}_{f} \longmapsto<\bar{F}>
$$

is plotted in a $\log -\log$ scale (see fig. III.11). As indicated in the previous section, $\langle\bar{F}\rangle$ represents an interaction force per particle. Fig. III.11 therefore shows that the force does not depend on the mean relative velocity only, but also on the solid fraction. As $\Phi$ increases, the force corresponding to a given velocity increases as well. It can be explained by considering the type of the flow around the particles. In the case of a dilute suspension, the flow around a particle is close to what it would be with a single particle in a infinite domain of fluid. As $\Phi$ reaches high values, the solid phase acts more like a porous medium: locally, the predominating phenomenon is a Poiseuille-like flow through inter-particle gaps. The latter phenomenon induces high viscous forces, which explains the fact that the force corresponding to concentrated suspension $(\Phi=0.28, N=100$ in Fig. III.11) can be one order of magnitude higher than the force corresponding to dilute suspension $(\Phi=0.003$, $N=1)$.

Those curves confirm the non-suitability of trivial interaction models (models based on interaction forces for a single particle) as soon as the suspension can no longer be considered dilute.

Remark:The relation which we obtain numerically results from a time averaging over a characteristic time $T$. It is therefore relevant only to model macroscopic phenomena with characteristic time much greater than $T$. 


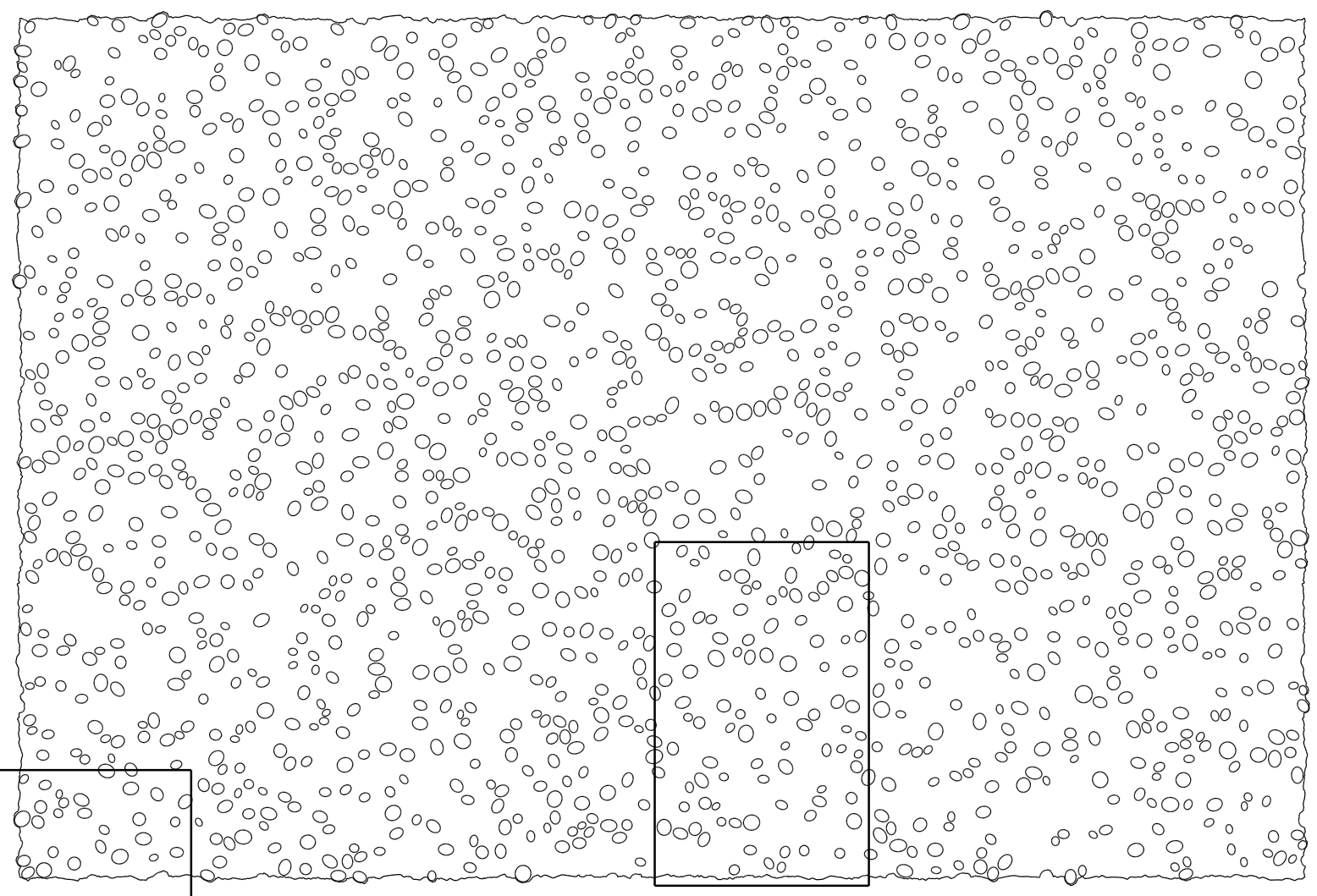

Figure III.3a: Mesh boundary.

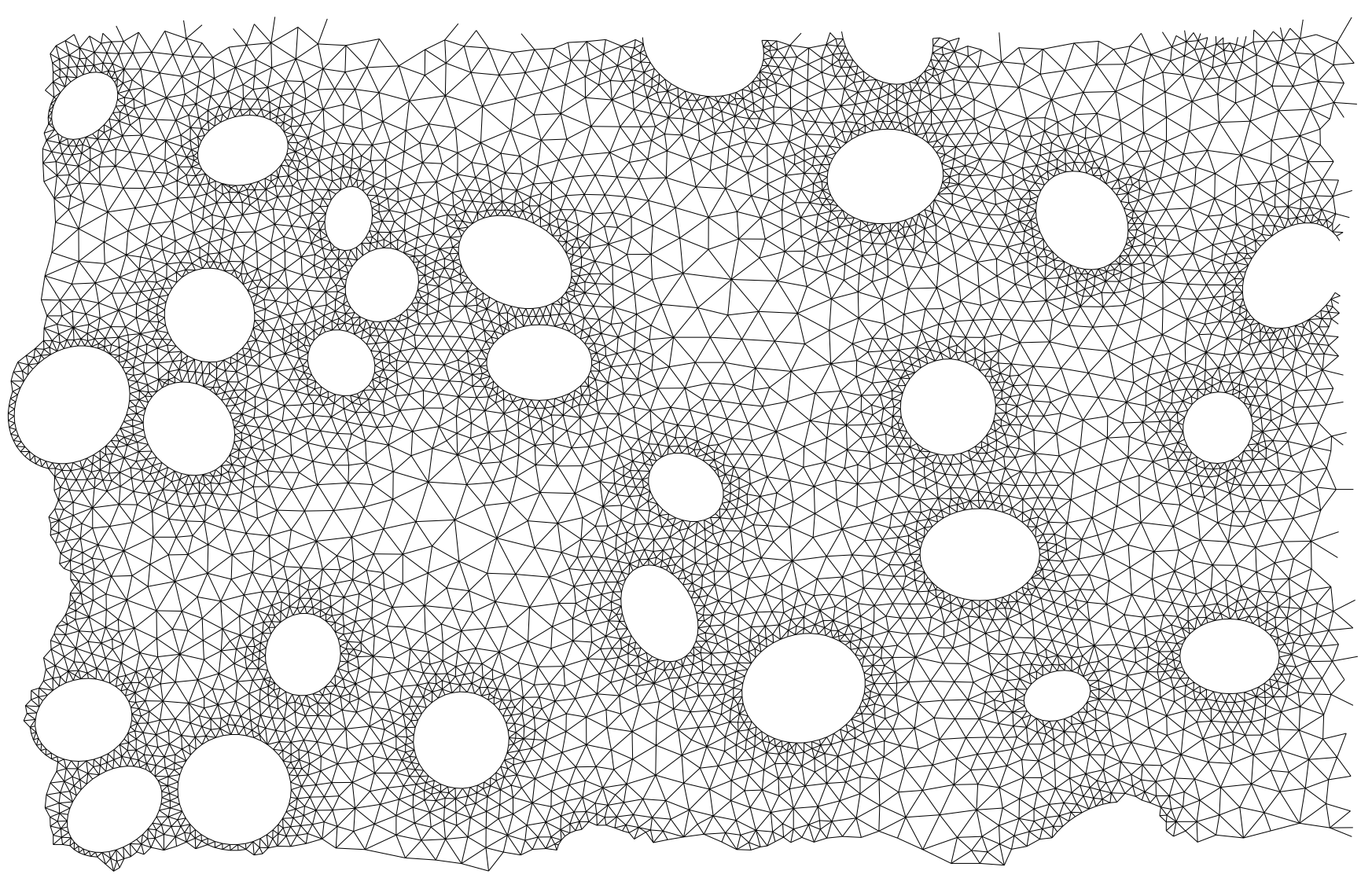

Figure III.3b: Mesh (detail). 


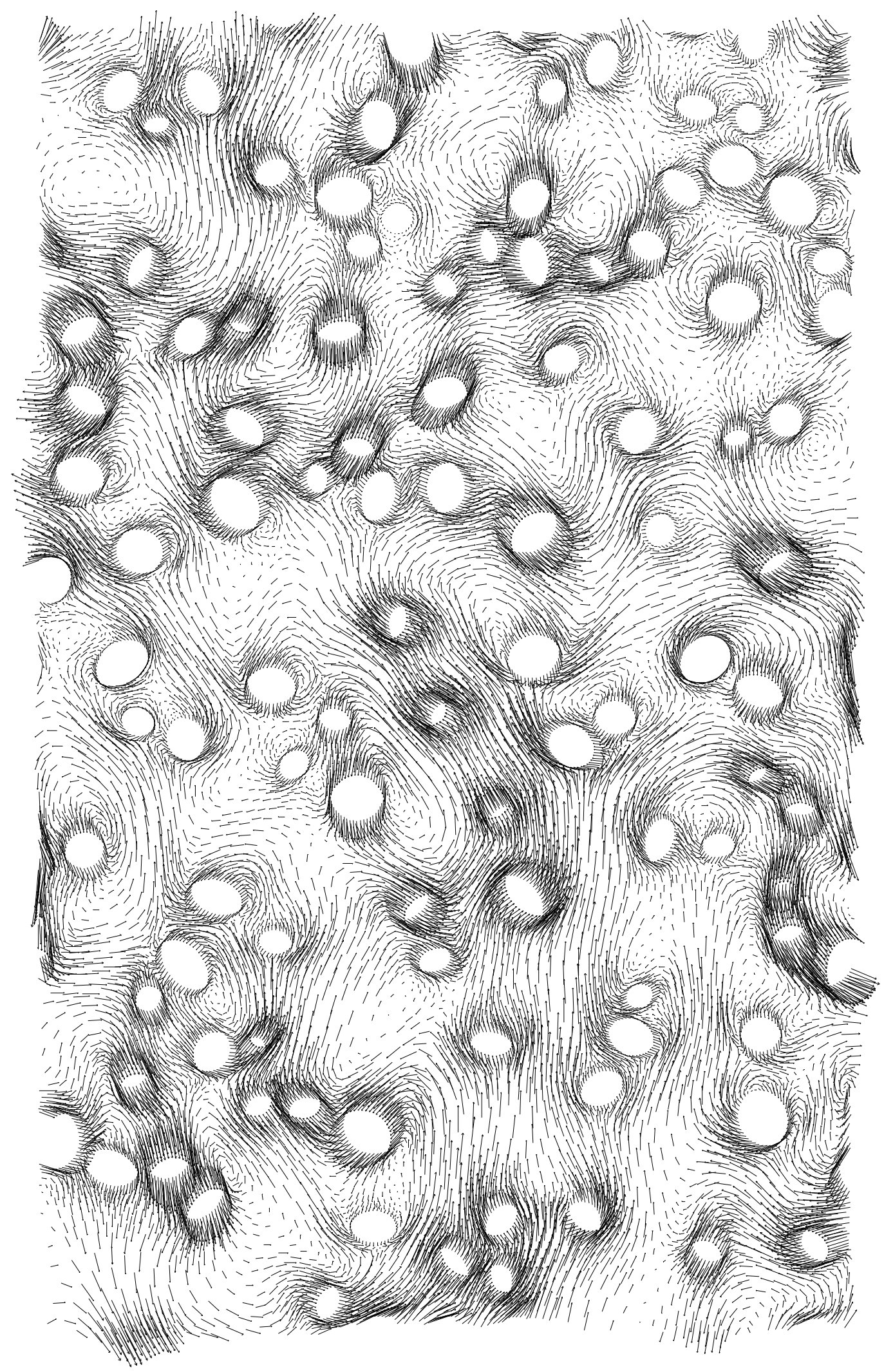

Figure III.4: Velocity field (detail). 


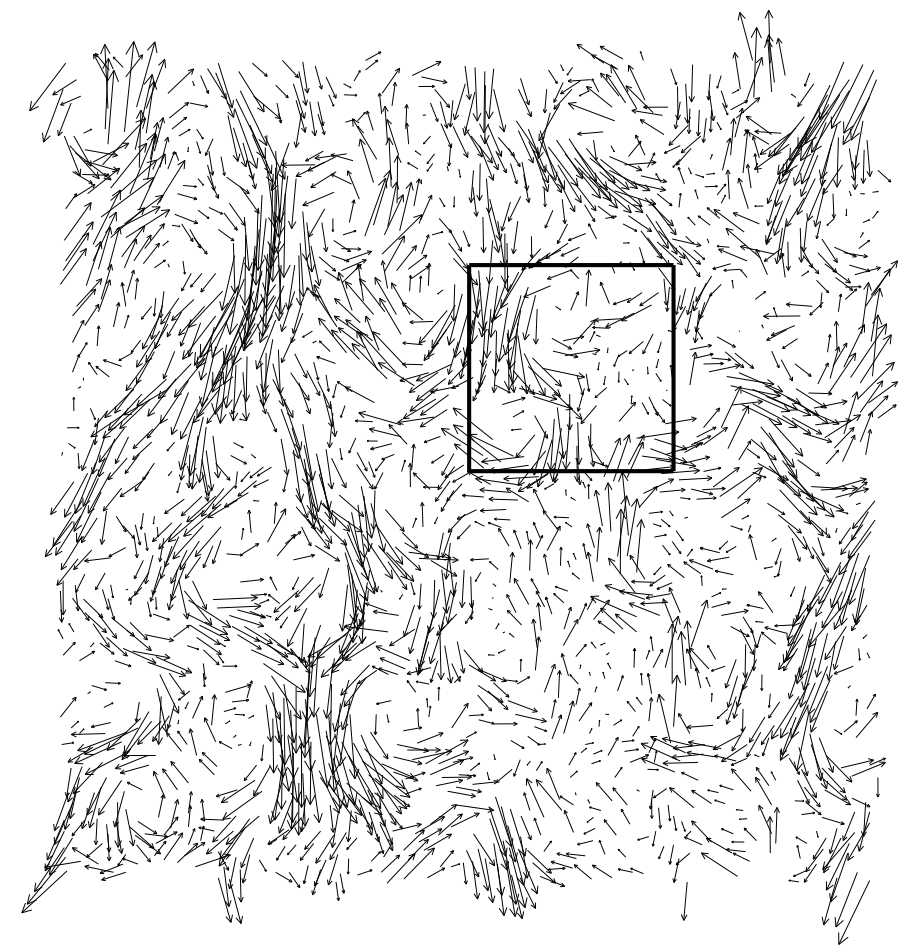

Figure III.5a: Particle velocity $(N=2000)$.

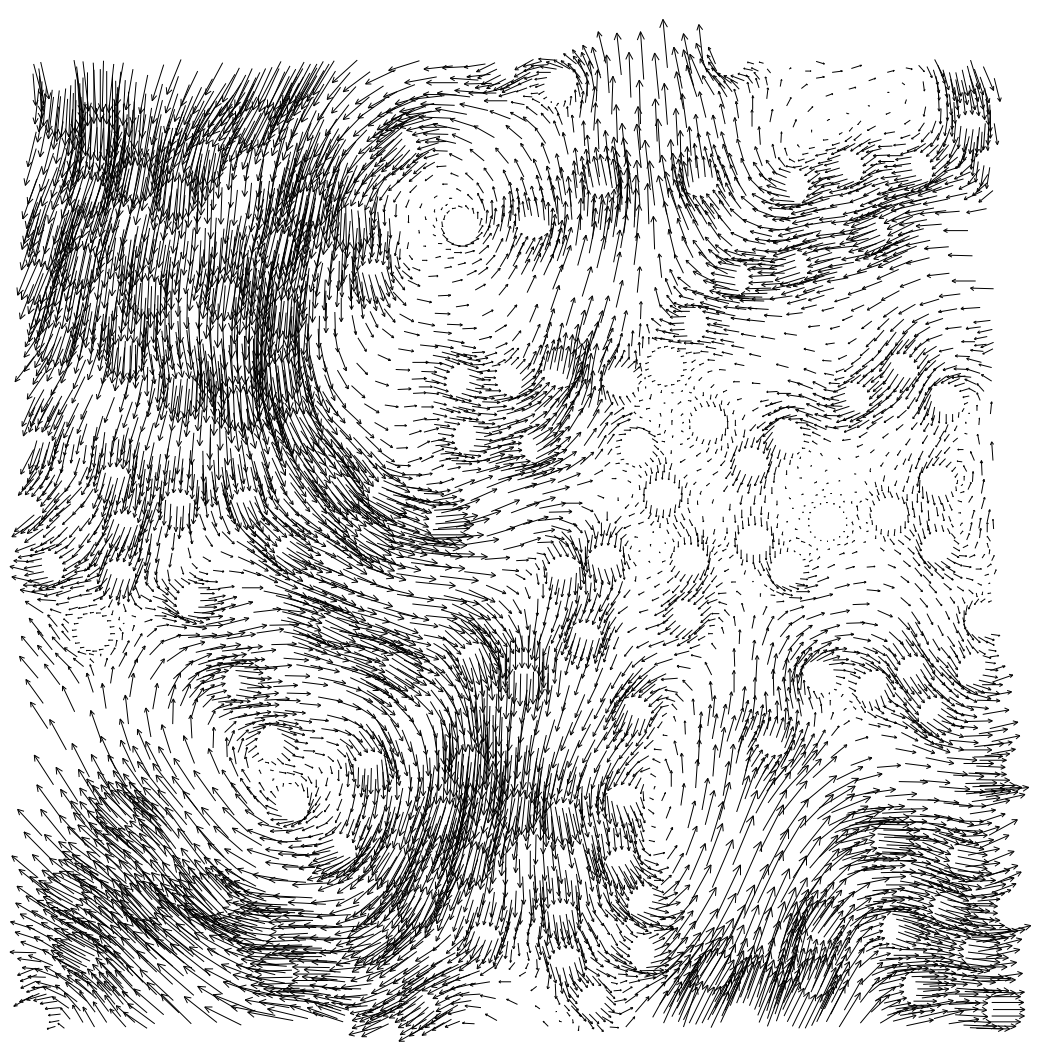

Figure III.5b: Fluid velocity (zoom). 


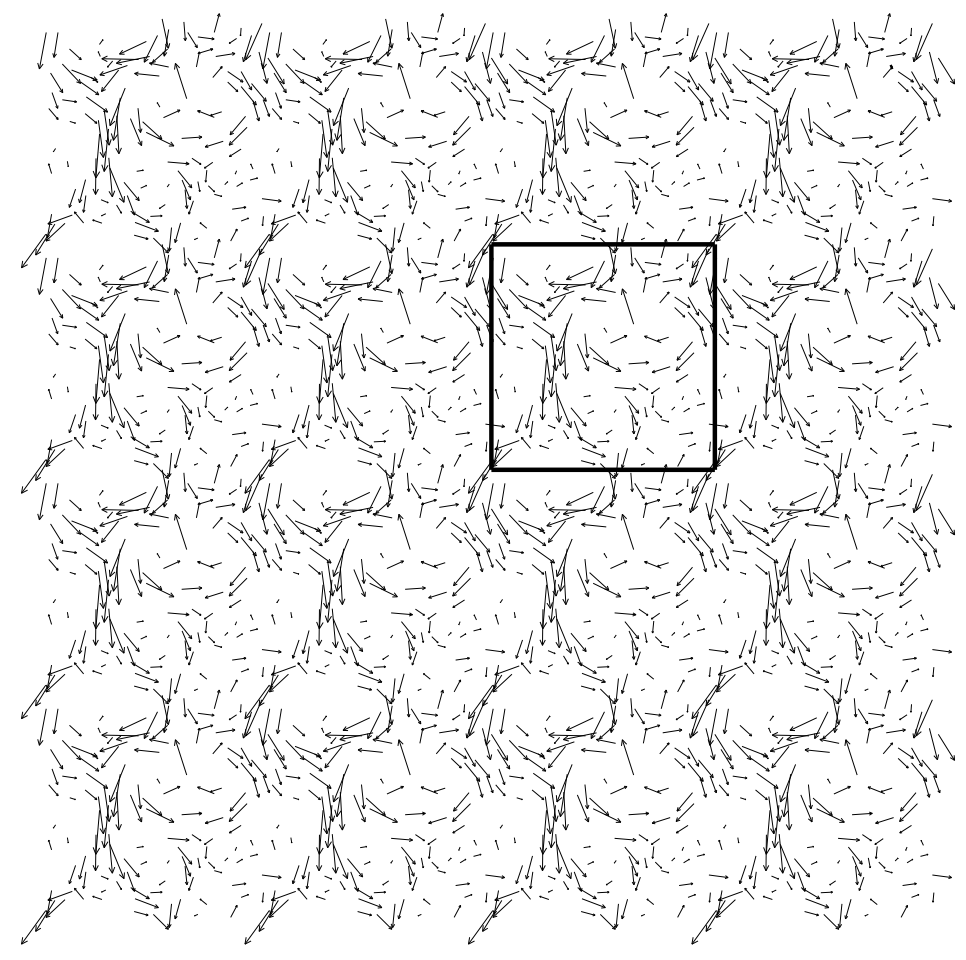

Figure III.6a: Particle velocity $(N=125 \times 16)$.

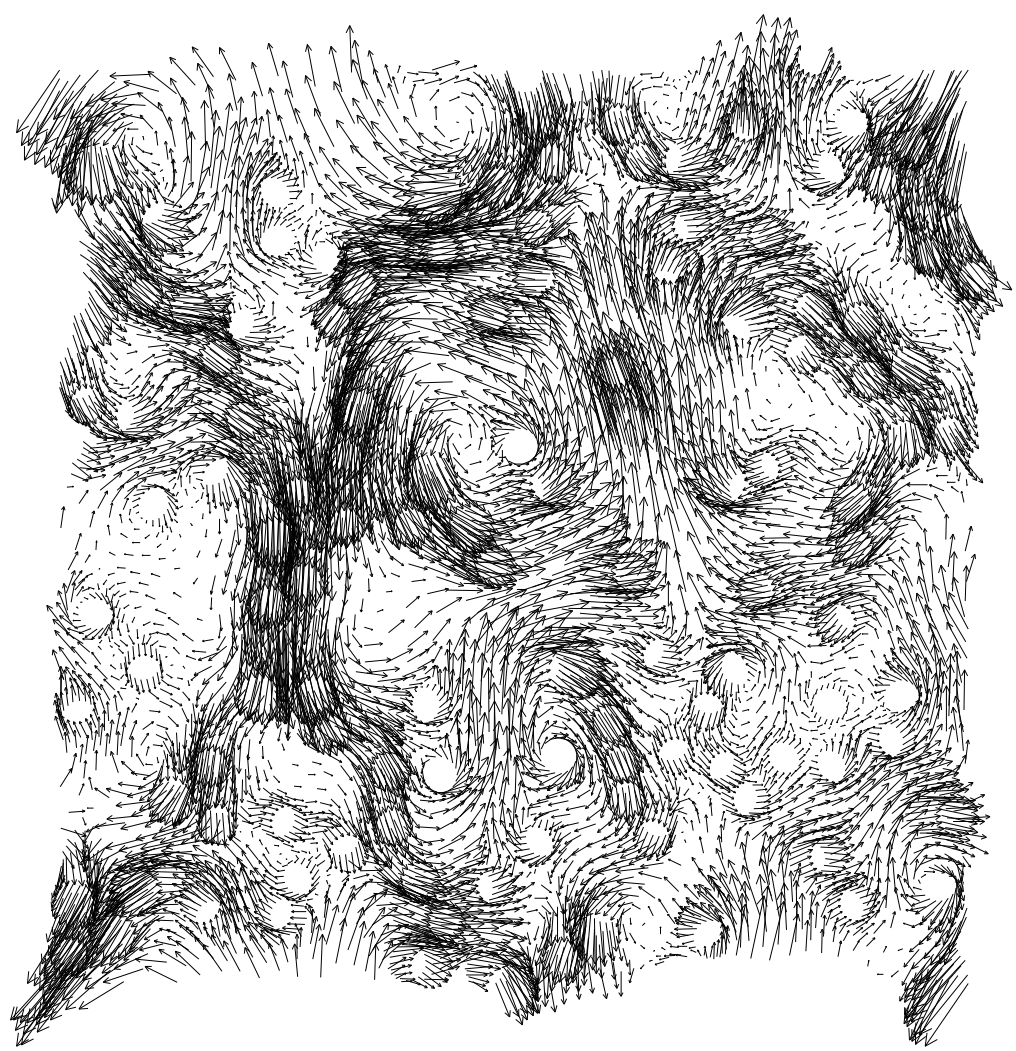

Figure III.6b: Fluid velocity. 


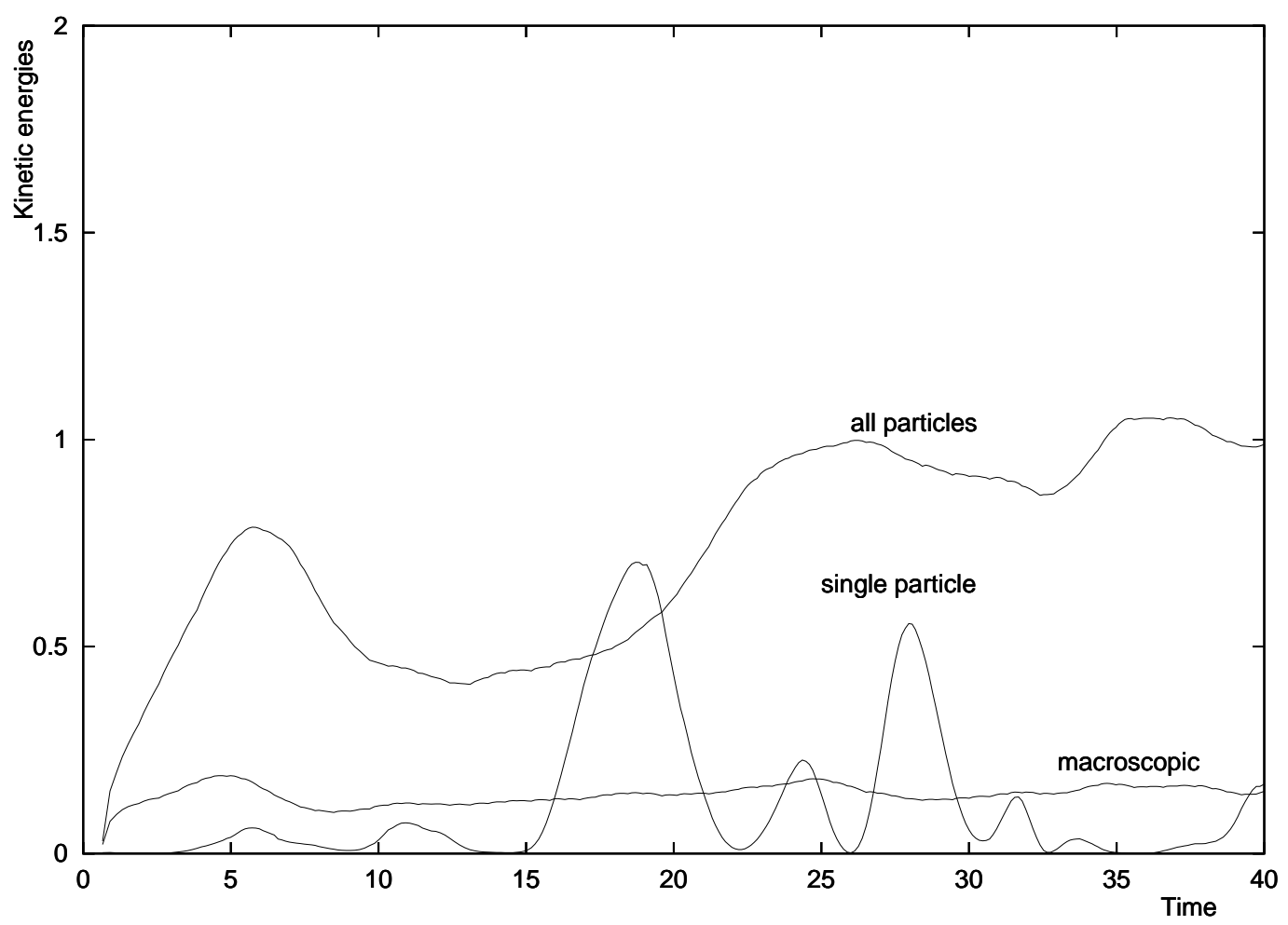

Figure III.7a: Kinetic energies, N=125.

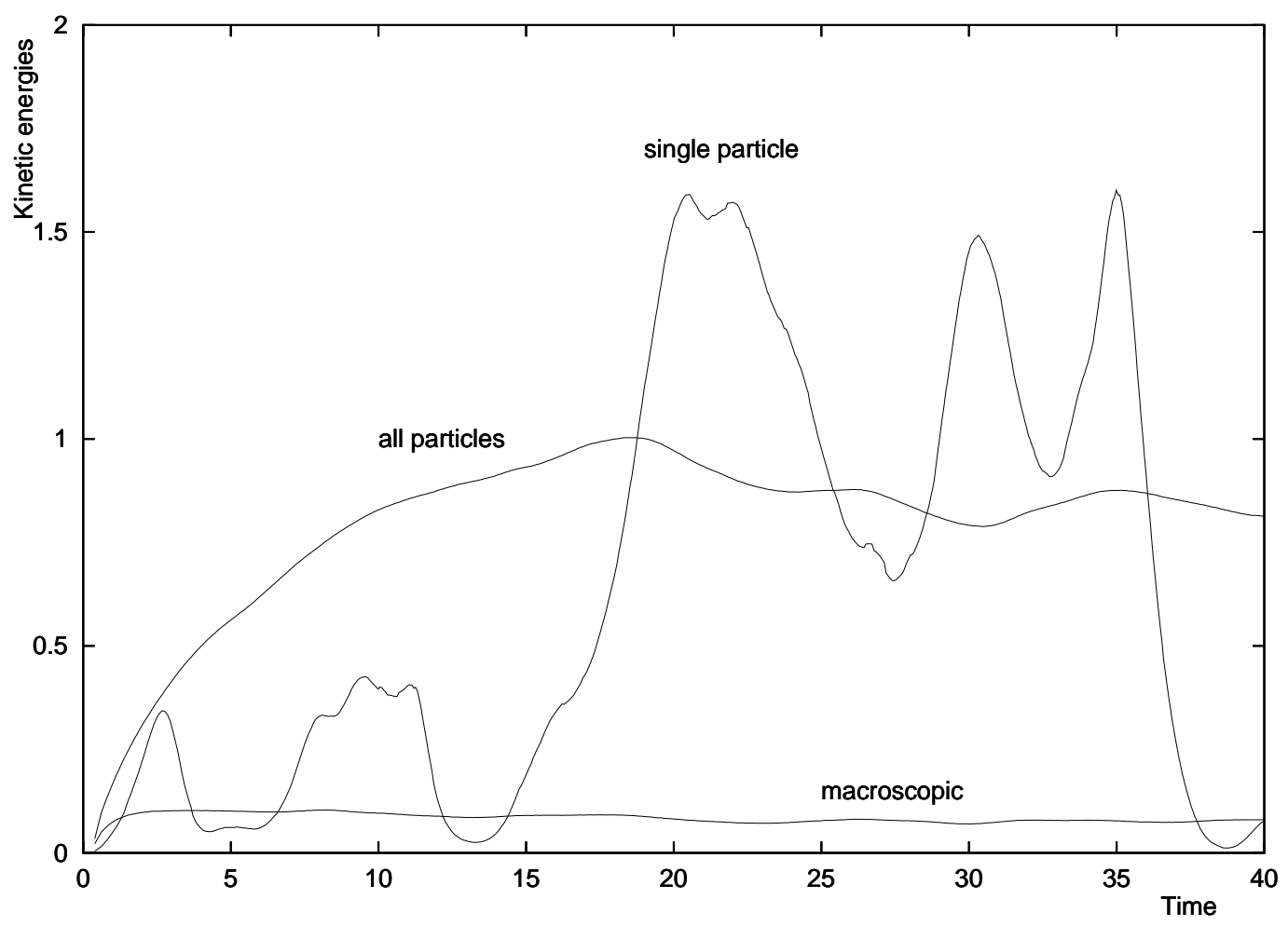

Figure III.7b: Kinetic energies, $\mathrm{N}=2000$; 


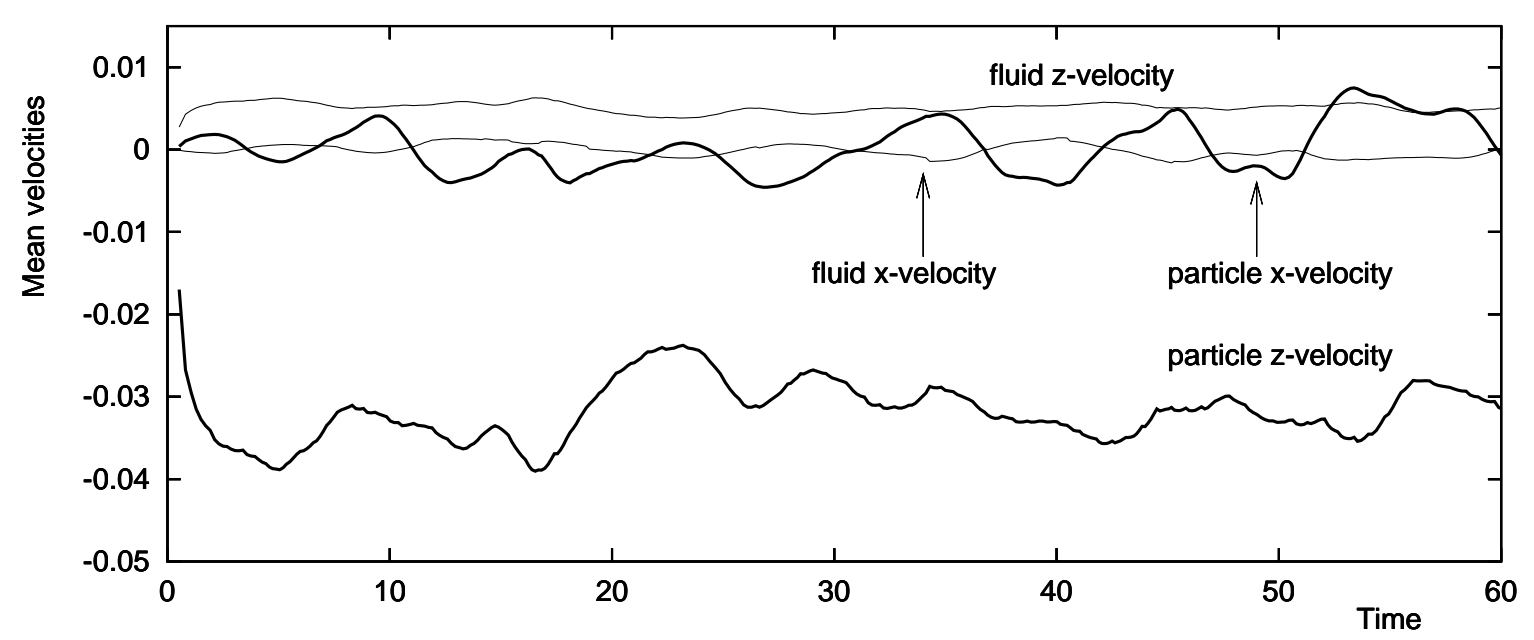

Figure III.8a: $\quad \mathrm{N}=50$.

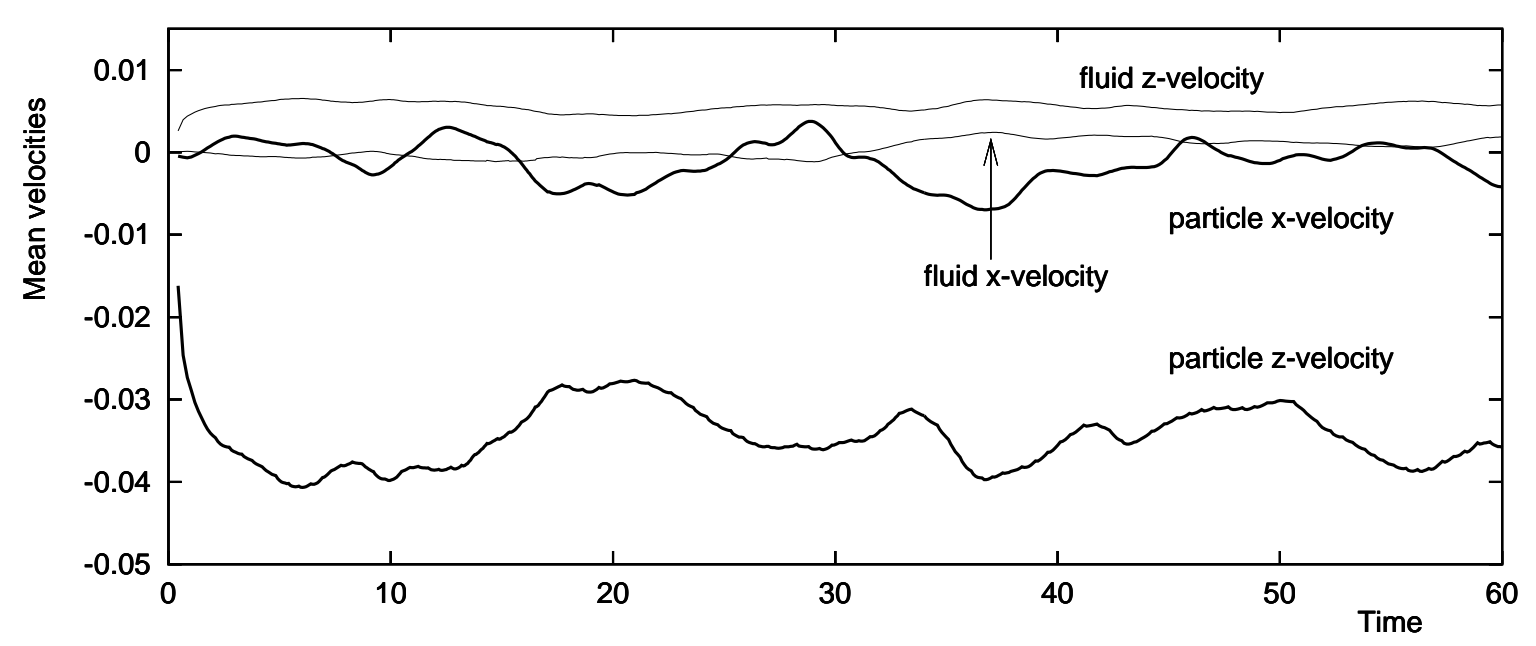

Figure III.8b: $\quad \mathrm{N}=200$.

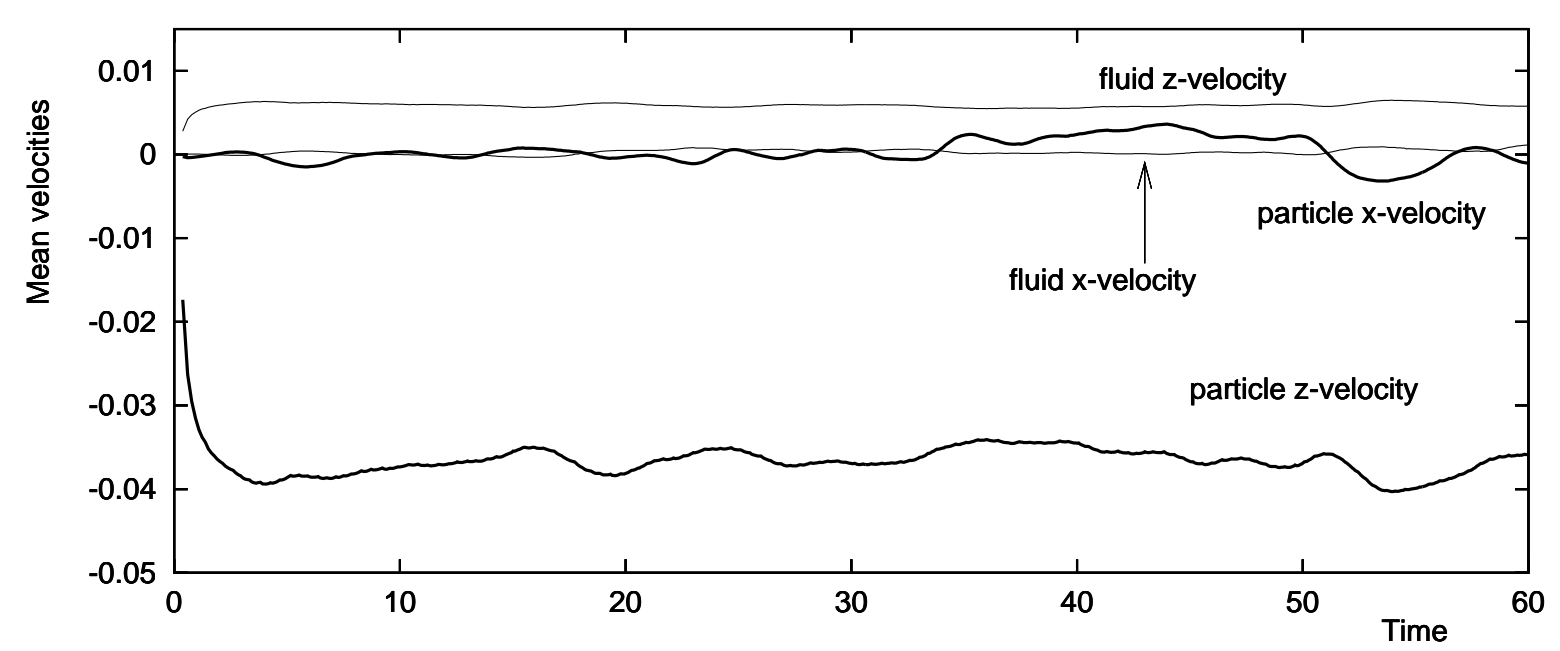

Figure III.8c: Average velocities, $\mathrm{N}=800$. 


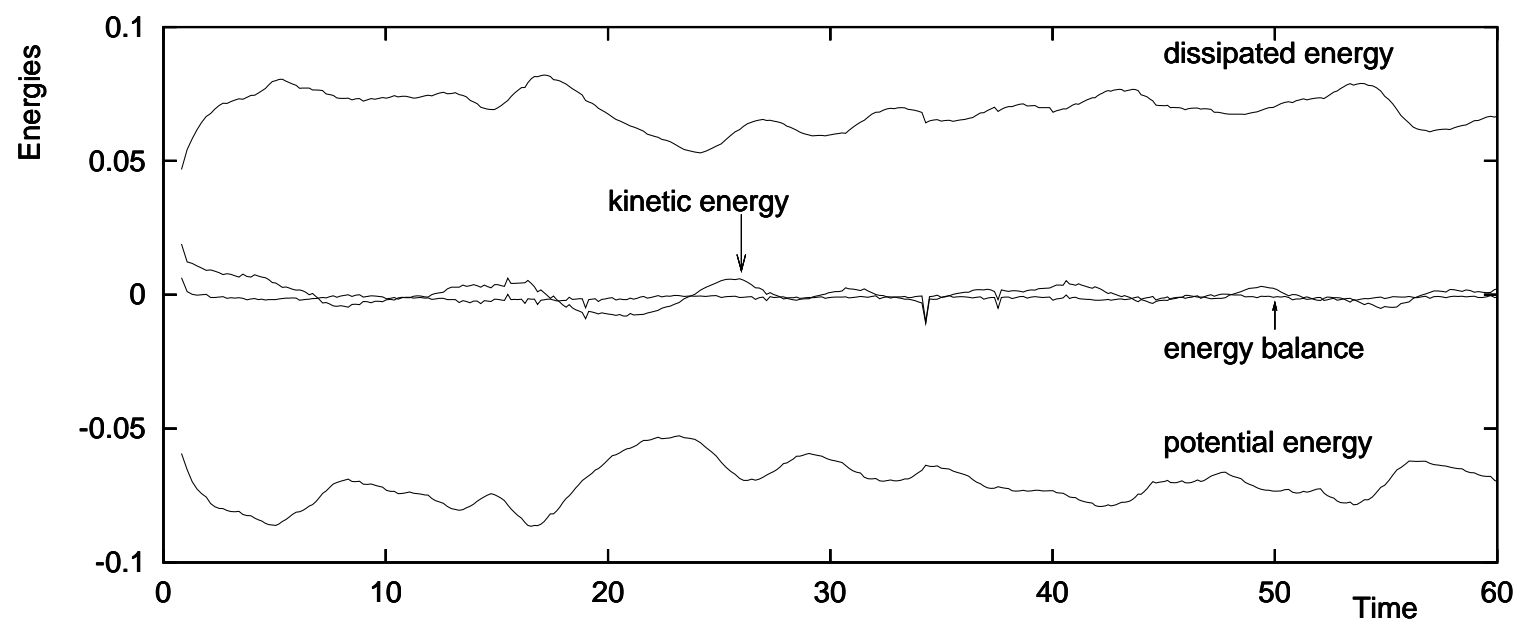

Figure III.9a: $\mathrm{N}=50$.

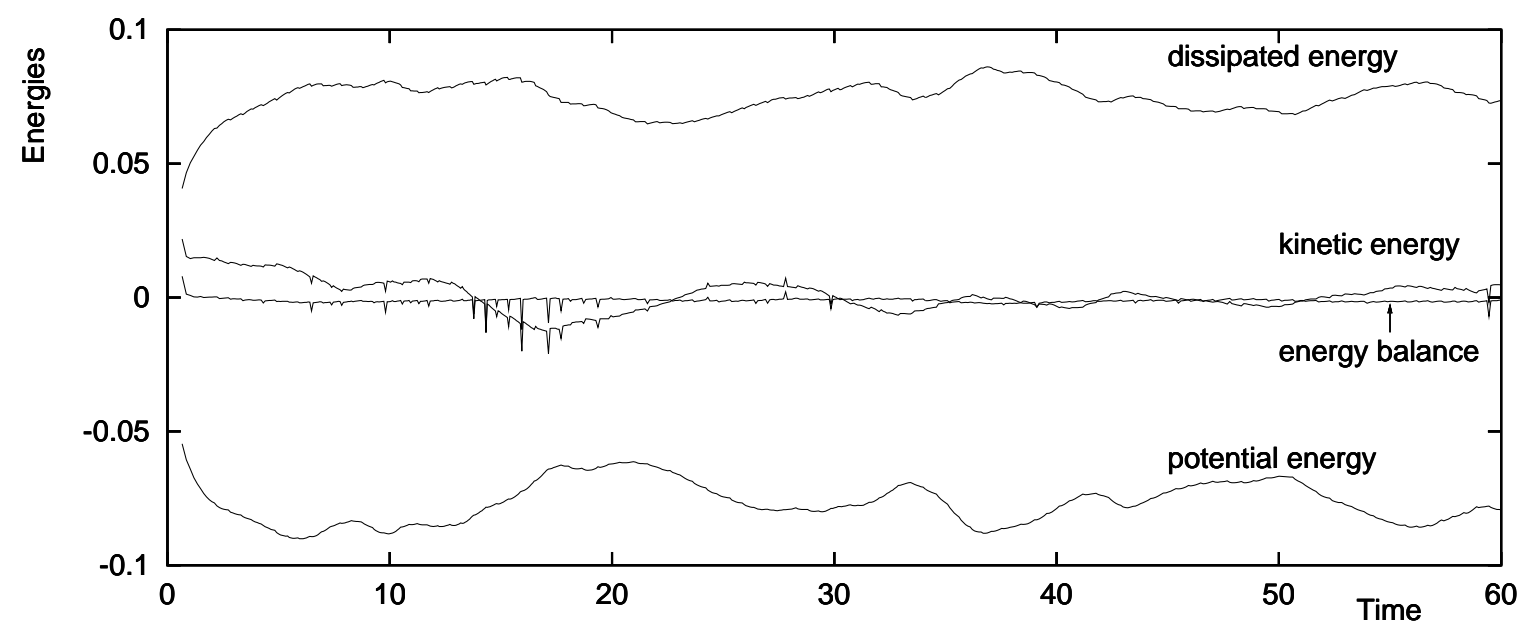

Figure III.9b: $\quad \mathrm{N}=200$.

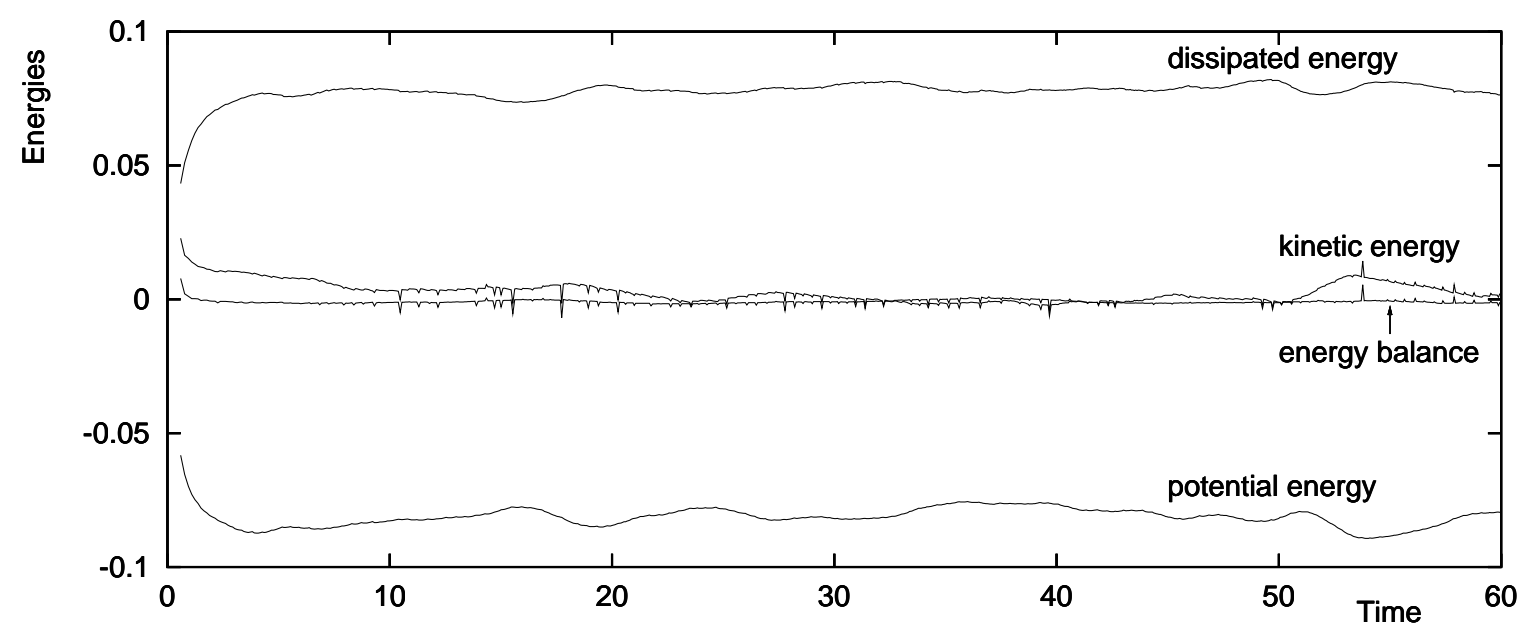

Figure III.9c: Energy balance, $\mathrm{N}=800$. 


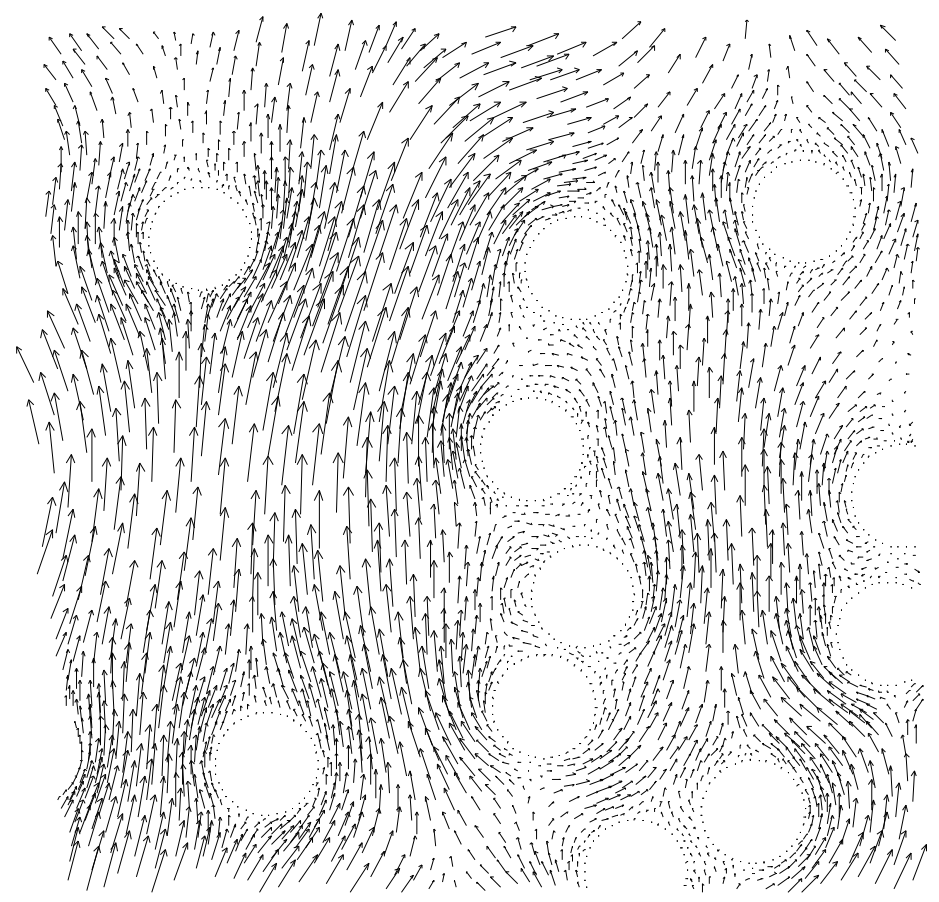

Figure III.10a: Relative velocity, $N=40, \operatorname{Re}_{p}=10$.

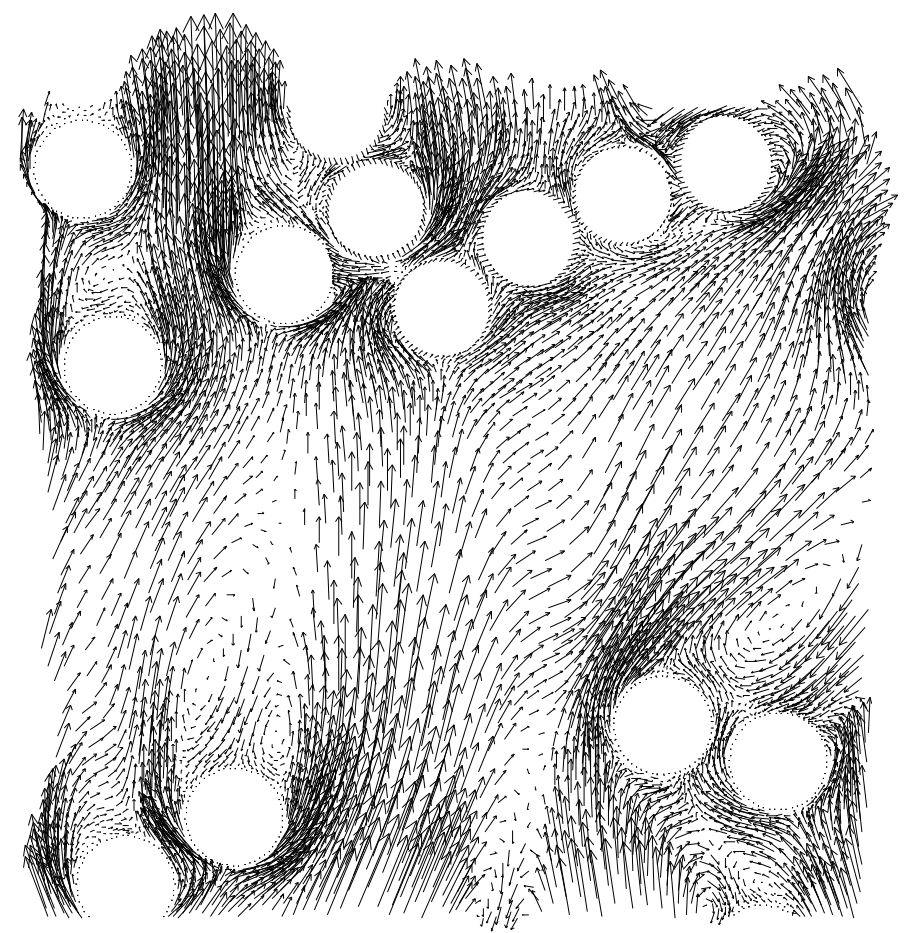

Figure III.10b: Relative velocity, $N=40, \operatorname{Re}_{p}=140$ (detail). 


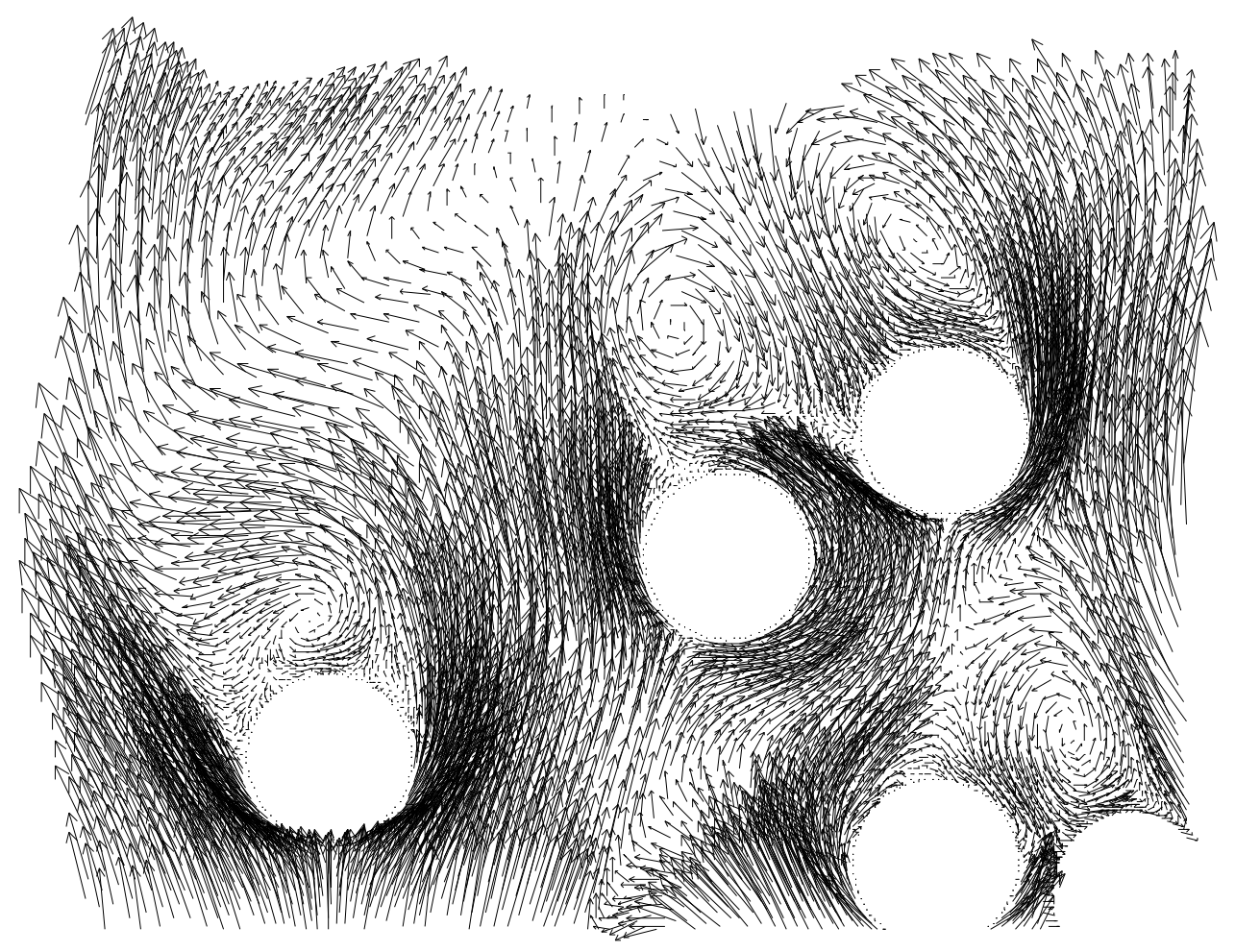

Figure III.10c: Relative velocity, $N=40, \operatorname{Re}_{p}=360$ (detail).

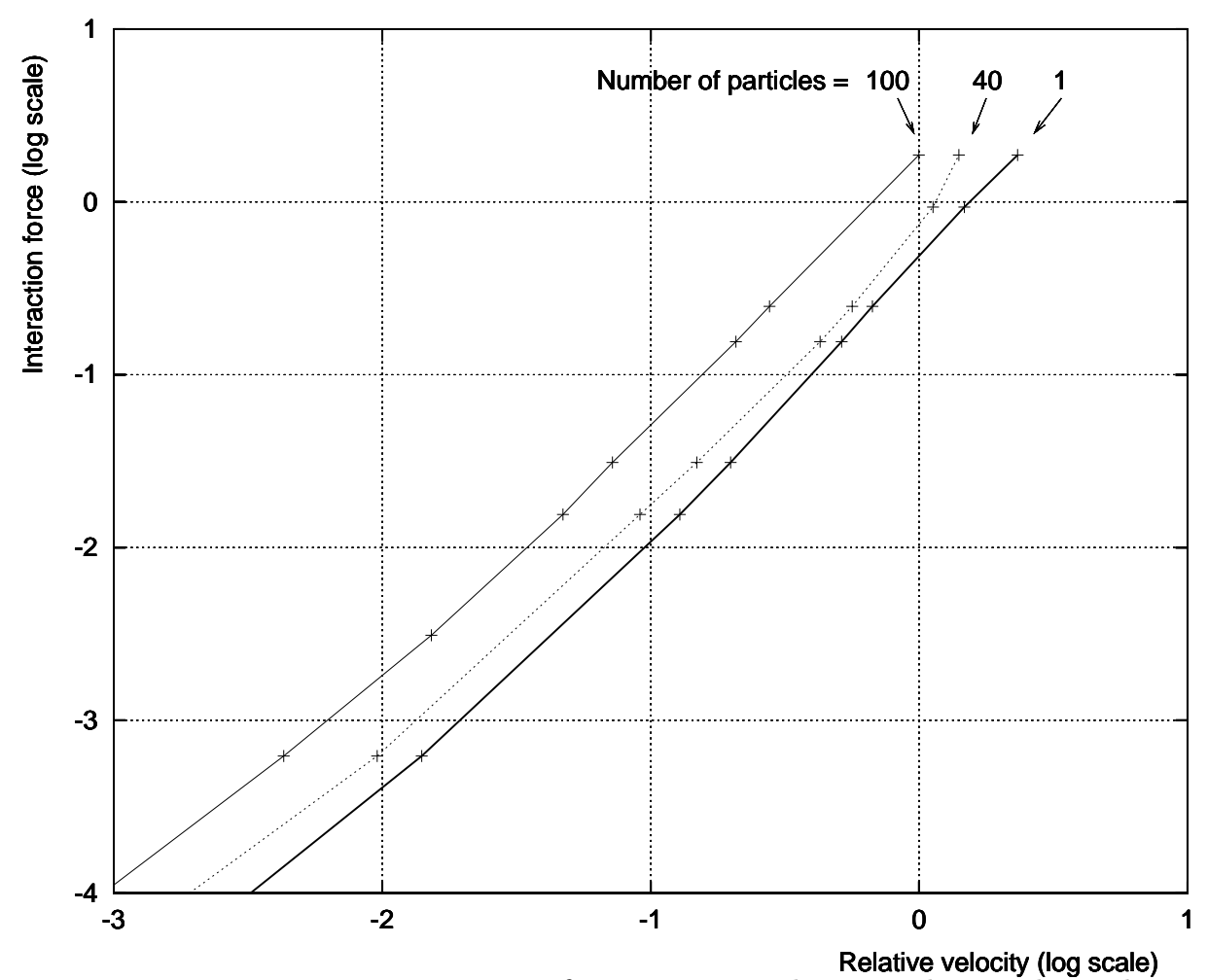

Figure III.11: Interaction force versus relative velocity, log - log scale. 


\section{Conclusion}

We presented a method to simulate the motion of particles in a viscous fluid. The main features of this method are:

- The advection term in the momentum equation for the fluid is taken into account by a method of characteristics. The time-discretized problem is then a generalized Stokes problem. As the resulting systems is symmetric, efficient methods like Preconditioned Conjugate Gradient can be used to compute velocity and pressure.

- This method is applicable to simulations in biperiodic domains. An extra unknown was introduced in order to take into account the vertical volume conservation. This new unknown plays the role of an extra pressure gradient, and it was proved it is a Lagrange multiplier for the vertical conservation constraint.

- A biperiodic mesh is built each time it is needed. As the computational mesh is biperiodic, periodic boundary conditions are simply prescribed by working on suitable Finite Element spaces.

- Numerical problems usually encountered in near-contact situations are suppressed by using a simple "cut-off"-like technique.

This method exhibits good stability properties, which permits long-time simulations of many-body motions (up to 5000 particles were simulated with this method). It presents good agreement with results obtained by other approaches. Furthermore, the computed energy balance reproduces a physically admissible behaviour. As experiments deal with spherical particles only (3D flow), strict validations based on them are unfortunately not possible for this $2 \mathrm{D}$ model.

The new capability to handle biperiodic Navier-Stokes flows makes it possible to study the long-time behaviour of mixtures with constant solid volume fraction, without boundary effects. Although the limits of biperiodic models still have to be established with more accuracy, this tool provides a promising way to investigate fluid-particle interaction phenomena.

\section{Acknowledgments}

We would like to acknowledge the helpful comments and suggestions of V. Girault, R. Glowinski, J.W. He, T. Hesla, H.H. Hu, D.D. Joseph, M. Knepley, Y. Kuznetsov, T. Pan, A. T. Patera, and B. Perthame. 


\section{References}

[1] G.K. Batchelor, Sedimentation in a Dilute Suspension of Spheres, J. Fluid Mech. 52, p.p. 245-268, 1972.

[2] F. Brezzi, On the Existence, Uniqueness and Approximation of Saddle-Point Problems Arising from Lagrangian Multipliers, R.A.I.R.O, $8^{e}$ année, R. 2, p.p. 129-151, août 1974.

[3] D.I. Dratler, W.R. Schowalter, Dynamic Simulation of Non-Brownian Hard Spheres, J. Fluid Mech. 325, p.p. 53-77, 1996.

[4] L. Durlofsky, J. F. Brady, G. Bossis, Dynamic Simulation of Hydrodynamically Interacting Particles, J. Fluid Mech. 180, p.p. 21-49, 1987.

[5] H. C. Elman, G. Golub, Inexact and Preconditioned Uzawa Algorithms for Saddle Point Problems, Siam Journal of Numerical Analysis 31, No 6, 1645-1661, 1994.

[6] R. Glowinski, T.-W. Pan, T. I. Hesla, and D.D. Joseph, A distributed Lagrange Multiplier/Fictitious Domain Method for Particulate Flows, Int. J. Multiphase Flow 25 (1999), pp. 755.

[7] Howard H. Hu, Direct Simulation of Flows of Solid-Liquid Mixtures, International Journal of Multiphase Flow 22, No 2, 335-352, 1996.

[8] T.J.R. Hughes, W. Liu, T.K. Zimmermann, Lagrangian-Eulerian Finite Element Formulation for Incompressible Viscous Flows, Comp. Methods in App. Mech. and Eng. 29, p.p. 329-349, 1981.

[9] A. A. Johnson, T. E. Tezduyar, Simulation of Multiple Spheres Falling in a LiquidFilled Tube, Computer Methods in Applied Mechanics and Engineering 134, p.p. 351-373, 1996.

[10] S. Kim S. and S. J. Karrila, Microhydrodynamics: Principles and Selected Applications, Butterworth-Heinemann, Boston, 1991.

[11] B. Maury, Characteristics ALE Method for the Unsteady 3D Navier-Stokes Equations with a Free Surface, International Journal of Computational Fluid Dynamics 6, p.p. 175-188, 1996.

[12] B. Maury and R. Glowinski, Fluid-Particle Flow: a Symmetric Formulation, C. R. Acad. Sci. Paris, t. 324, Série I, pp. 1079-1084, 1997.

[13] B. Maury, A Many-Body Lubrication Model, C. R. Acad. Sci. Paris, t. 325, Série I, pp. 1053-1058, 1997.

[14] M. Pedercini, A. T. Patera,M. E. Cruz, Variational Bound Finite Element Methods for Three-Dimensional Creeping Porous-Media and Sedimentation Flows, International Journal for Numerical methods in Fluids, vol. 26, p.p. 145-175 (1998).

[15] O. Pironneau, J. Liou, T. Tezduyar, Characteristic-Galerkin and Galerkin Least Squares Space-Time Formulations for the Advection-Diffusion Equation with TimeDependent Domains, Computer Method in Applied Mechanics and Engineering 100, 117-141, 1992. 
[16] A. Prosperetti, D. Z. Zhang, Finite-Particle-Size Effects in Disperse Two-Phase Flows, Theoret. Comput. Fluid Dynamics, 7, p.p. 429-440, 1995.

[17] A.S. Sangani, A. K. DidWania, Dynamic Simulations of Flows of Bubbly Liquids at Large Reynolds Numbers, Journal of Fluid Mechanics, 250, p.p. 307-337, 1993.

[18] A.S. Sangani, A. Prosperetti, Numerical Simulation of the motion of Particles at Large Reynolds numbers, Particulate Two-Phase Flow, Edited by M.C. Roco, p.p. 971-998, Butterworth-Heinamann, New York, 1993.

[19] T. Sato, S. M. Richardson, Numerical Simulation Method for Viscoelastic Flows with Free Surfaces - Fringe Element Generation Method, International Journal for Numerical Methods in Fluids, 19, p.p. 555-574, 1994.

[20] P. Singh, D.D. Joseph, Dynamics of Fluidized Suspensions of Spheres of Finite Sizes, International Journal of Multiphase Flows, 21, p.p. 1-26, 1995. 